\title{
Initial-Boundary Value Problems for Multi-Term Time-Fractional Diffusion Equations with Positive Constant Coefficients*
}

\author{
Zhiyuan $\mathrm{LI}^{\dagger} \quad$ Yikan LIU $^{\dagger} \quad$ Masahiro YAMAMOTO ${ }^{\dagger}$
}

\begin{abstract}
In this paper, we investigate the well-posedness and the long-time asymptotic behavior for the initial-boundary value problem for multi-term time-fractional diffusion equations, where the time differentiation consists of a finite summation of Caputo derivatives with decreasing orders in $(0,1)$ and positive constant coefficients. By exploiting several important properties of multinomial Mittag-Leffler functions, various estimates follow from the explicit solutions in form of these special functions. Then the uniqueness and continuous dependency upon initial value and source term are established, from which the continuous dependence of solution of Lipschitz type with respect to various coefficients is also verified. Finally, by a Laplace transform argument, it turns out that the decay rate of the solution as $t \rightarrow \infty$ is dominated by the minimum order of the time-fractional derivatives.
\end{abstract}

Keywords Initial-boundary value problem, Time-fractional diffusion equation, Multinomial Mittag-Leffler function, Well-posedness,

Long-time asymptotic behavior, Laplace transform

AMS subject classifications 35G16, 35R11, 33E12, 35B40, 44A10

\section{Introduction}

Let $\Omega$ be an open bounded domain in $\mathbb{R}^{d}$ with a smooth boundary (for example, of $C^{2}$ class) and $T>0$ be fixed arbitrarily. For a fixed positive integer $m$, let $\alpha_{j}$ and $q_{j}(j=1, \ldots, m)$ be positive constants such that $1>\alpha_{1}>\cdots>\alpha_{m}>0$. Consider the following initial-boundary value problem for the multi-term time-fractional diffusion equation

$$
\begin{cases}\sum_{j=1}^{m} q_{j} \partial_{t}^{\alpha_{j}} u(x, t)=L u(x, t)+F(x, t), & x \in \Omega, 0<t \leq T, \\ u(x, t)=0, & x \in \partial \Omega, 0<t \leq T, \\ u(x, 0)=a(x), & x \in \Omega,\end{cases}
$$

where $L$ is a symmetric uniformly elliptic operator with the homogeneous Dirichlet boundary condition, and we can assume $q_{1}=1$ without lose of generality. The regularities of the initial value $a$ and the source term $F$ will be specified later. Here $\partial_{t}^{\alpha_{j}}$ denotes the Caputo derivative

\footnotetext{
Manuscript last updated: October 2, 2018.

$\dagger$ Graduate School of Mathematical Sciences, the University of Tokyo, 3-8-1 Komaba, Meguro-ku,

Tokyo 153-8914, Japan.

E-mail: zyli@ms.u-tokyo.ac.jp, ykliu@ms.u-tokyo.ac.jp, myama@ms.u-tokyo.ac.jp

*This work was supported by the Program for Leading Graduate Schools, MEXT, Japan.
} 
defined by

$$
\partial_{t}^{\alpha_{j}} f(t):=\frac{1}{\Gamma\left(1-\alpha_{j}\right)} \int_{0}^{t} \frac{f^{\prime}(s)}{(t-s)^{\alpha_{j}}} \mathrm{~d} s
$$

where $\Gamma(\cdot)$ is a usual Gamma function. For various properties of the Caputo derivative, we refer to Kilbas et al. [12] and Podlubny [24]. See also [8,28] for further contents on fractional calculus.

In the case of $m=1$, equation (1.1) is reduced to its single-term counterpart

$$
\partial_{t}^{\alpha} u=L u+F \quad \text { in } \Omega \times(0, T], \alpha \in(0,1) .
$$

The above formulation has been studied extensively from different aspects due to its vast capability of modeling the anomalous diffusion phenomena in highly heterogeneous aquifer and complex viscoelastic material (see Adams \& Gelhar [1, Ginoa et al. 6], Hatano \& Hatano [9], Nigmatullin [22] and the references therein). Indeed, although the single-term time-fractional diffusion equation inherits certain properties from the diffusion equation with time derivative of natural number order, it differs considerably from the traditional one especially in sense of its limited smoothing effect in space and slow decay in time. In Luchko [16, a maximum principle of the initial-boundary value problem for (1.4) was established, and the uniqueness of a classical solution was proved. Luchko [17] represented the generalized solution to (1.4) with $F=0$ by means of the Mittag-Leffler function and gave the unique existence result. Sakamoto \& Yamamoto [26] carried out a comprehensive investigation including the well-posedness of the initial-boundary value problem for (1.4) as well as the long-time asymptotic behavior of the solution. It turns out that the spatial regularity of the solution is only moderately improved from that of the initial value, and the solution decays with order $t^{-\alpha}$ as $t \rightarrow \infty$. Recently, the Lipschitz stability of the solution to (1.4) with respect to $\alpha$ and the diffusion coefficient was proved as a byproduct of an inverse coefficient problem in Li et al. [13]. For other discussions concerning equation (1.4), see e.g., Gorenflo et al. [7] and Luchko [15], Prüss [25]. Regarding numerical treatments, we refer to Liu et al. [14] and Meerschaert \& Tadjeran [20] for the finite difference method and Jin et al. [1] for the finite element method.

As natural extension, equation (1.1) is expected to improve the modeling accuracy in depicting the anomalous diffusion due to its potential feasibility. However, to the authors' best knowledge, published works on this extension are quite limited in spite of rich literatures on its single-term version. Luchko [18] developed the maximum principle for problem (1.1)-1.3) and constructed a generalized solution when $F=0$ by means of the multinomial Mittag-Leffler functions. Jiang et al. [10] considered fractional derivatives in both time and space and derived analytical solutions. As for the asymptotic behavior, for $m=2$ it reveals in Mainardi et al. 21] that the dominated decay rate of the solution is related to the minimum order of time fractional derivative. On the other hand, Beckers \& Yamamoto [4 investigated (1.1)-1.3) in a slightly more general formulation and obtained a weaker regularity result than that in [26].

In this paper, we are concerned with the well-posedness and the long-time asymptotic behavior of the solution to the initial-boundary value problem (1.1)-(1.3), and we attempt to establish results parallel to that for the single-term case. On basis of the explicit representation of the solution, we give estimates for the solution by exploiting several properties of the multinomial Mittag-Leffler function. Moreover, as long as the continuous dependency of the solution 
on the initial value and the source term is verified, we can also deduce the Lipschitz stability of the solution to (1.1)-(1.3) with respect to $\alpha_{j}, q_{j}(j=1, \ldots, m)$ and the diffusion coefficient immediately. For the long-time asymptotic behavior, we employ the Laplace transform in time to show that the decay rate as $t \rightarrow \infty$ is indeed $t^{-\alpha_{m}}$, where $\alpha_{m}$ is the minimum order of Caputo derivatives in time.

The rest of this paper is organized as follows. The main results concerning problem (1.1)(1.3) are collected in Section 2, which includes theorems on well-posedness and long-time asymptotic behavior of the solution. The proofs of the main theorems are postponed to Section 3 which is further divided into three subsections. Subsection 3.1 is devoted to a close scrutiny of the multinomial Mittag-Leffler functions, which serves as essential keys in the proofs of well-posedness results in Subsection 3.2. Due to the difference of techniques, the asymptotic behavior is proved independently in Subsection 3.3 Finally, concluding remarks are given in Section 4

\section{Main Results}

In this section, we state the main results obtained in this paper. More precisely, we give a priori estimates for the solution $u$ to (1.1)-(1.3) with respect to the initial value (Theorem 2.1), the source term (Theorem 2.2), and Lipschitz continuous dependence of the solutions on coefficients and orders (Theorem 2.3) so that stability and uniqueness follow, and we describe the asymptotic behavior of the solution in Theorem 2.4

To this end, we first fix some general settings and notations. Let $L^{2}(\Omega)$ be a usual $L^{2}$ space with the inner product $(\cdot, \cdot)$ and $H_{0}^{1}(\Omega), H^{2}(\Omega)$ denote the Sobolev spaces (see, e.g., Adams [2]). The elliptic operator $L$ is defined for $f \in \mathcal{D}(-L):=H^{2}(\Omega) \cap H_{0}^{1}(\Omega)$ as

$$
L f(x)=\sum_{i, j=1}^{d} \partial_{j}\left(a_{i j}(x) \partial_{i} f(x)\right)+c(x) f(x), \quad x \in \Omega,
$$

where $a_{i j}=a_{j i}(1 \leq i, j \leq d)$ and $c \leq 0$ in $\bar{\Omega}$. Moreover, it is assumed that $a_{i j} \in C^{1}(\bar{\Omega})$, $c \in C(\bar{\Omega})$ and there exists a constant $\delta>0$ such that

$$
\delta \sum_{i=1}^{d} \xi_{i}^{2} \leq \sum_{i, j=1}^{d} a_{i j}(x) \xi_{i} \xi_{j}, \quad \forall x \in \bar{\Omega}, \forall\left(\xi_{1}, \ldots, \xi_{d}\right) \in \mathbb{R}^{d} .
$$

On the other hand, let $\left\{\lambda_{n}, \varphi_{n}\right\}_{n=1}^{\infty}$ be the eigensystem of the elliptic operator $-L$ such that $0<\lambda_{1}<\lambda_{2} \leq \cdots, \lambda_{n} \rightarrow \infty$ as $n \rightarrow \infty$ and $\left\{\varphi_{n}\right\} \subset H^{2}(\Omega) \cap H_{0}^{1}(\Omega)$ forms an orthonormal basis of $L^{2}(\Omega)$. Then the fractional power $(-L)^{\gamma}$ is well-defined for $\gamma \in \mathbb{R}$ (see Pazy [23]) with

$$
\mathcal{D}\left((-L)^{\gamma}\right)=\left\{f \in L^{2}(\Omega) ; \sum_{n=1}^{\infty}\left|\lambda_{n}^{\gamma}\left(f, \varphi_{n}\right)\right|^{2}<\infty\right\},
$$

and $\mathcal{D}\left((-L)^{\gamma}\right)$ is a Hilbert space with the norm

$$
\|f\|_{\mathcal{D}\left((-L)^{\gamma}\right)}=\left(\sum_{n=1}^{\infty}\left|\lambda_{n}^{\gamma}\left(f, \varphi_{n}\right)\right|^{2}\right)^{1 / 2} .
$$


Also we note that $\mathcal{D}\left((-L)^{\gamma}\right) \subset H^{2 \gamma}(\Omega)$ for $\gamma>0$ and especially $\mathcal{D}\left((-L)^{1 / 2}\right)=H_{0}^{1}(\Omega)$.

Now we are well-prepared to consider the dependency of the solution $u$ to the initialboundary value problem (1.1)-(1.3) upon the initial value $a$ and the source term $F$. In view of the superposition principle, it suffices to deal with the cases $F=0, a \neq 0$ and $a=0, F \neq 0$ separately.

Theorem 2.1 Let $F=0,0 \leq \gamma \leq 1$ and $a \in \mathcal{D}\left((-L)^{\gamma}\right)$, where we interpret $\frac{1}{1-\gamma}=\infty$ if $\gamma=1$. Concerning the solution $u$ to the initial-boundary value problem (1.1)-(1.3), the followings hold true.

(a) There exists a unique solution $u \in C\left([0, T] ; L^{2}(\Omega)\right) \cap C\left((0, T] ; H^{2}(\Omega) \cap H_{0}^{1}(\Omega)\right)$ to (1.1)(1.3). Actually, $u \in L^{\frac{1}{1-\gamma}}\left(0, T ; H^{2}(\Omega) \cap H_{0}^{1}(\Omega)\right)$ and there exists a constant $C>0$ such that

$$
\begin{aligned}
& \|u\|_{C\left([0, T] ; L^{2}(\Omega)\right)} \leq C\|a\|_{L^{2}(\Omega)}, \\
& \|u(\cdot, t)\|_{H^{2}(\Omega)} \leq C\|a\|_{\mathcal{D}\left((-L)^{\gamma}\right)} t^{\alpha_{1}(\gamma-1)}, \quad 0<t \leq T .
\end{aligned}
$$

(b) We have

$$
\lim _{t \rightarrow 0}\|u(\cdot, t)-a\|_{\mathcal{D}\left((-L)^{\gamma}\right)}=0 .
$$

(c) There holds $\partial_{t} u \in C\left((0, T] ; L^{2}(\Omega)\right)$. Moreover, there exists a constant $C>0$ such that

$$
\left\|\partial_{t} u(\cdot, t)\right\|_{L^{2}(\Omega)} \leq C\|a\|_{\mathcal{D}\left((-L)^{\gamma}\right)} t^{\alpha_{1} \gamma-1}, \quad 0<t \leq T .
$$

(d) If $\gamma>0$, then $\partial_{t}^{\beta} u \in L^{\frac{1}{1-\gamma}}\left(0, T ; L^{2}(\Omega)\right)$ for $0<\beta \leq \alpha_{1}$. Moreover, for $0<\beta<1$, there exists a constant $C>0$ such that

$$
\left\|\partial_{t}^{\beta} u(\cdot, t)\right\|_{L^{2}(\Omega)} \leq C\|a\|_{\mathcal{D}\left((-L)^{\gamma}\right)} t^{\alpha_{1} \gamma-\beta}, \quad 0<t \leq T .
$$

Theorem 2.2 Let $a=0,1 \leq p \leq \infty, 0 \leq \gamma \leq 1$ and $F \in L^{p}\left(0, T ; \mathcal{D}\left((-L)^{\gamma}\right)\right)$, where we interpret $1 / p=0$ if $p=\infty$. Concerning the solution $u$ to the initial-boundary value problem (1.1)-(1.3), the followings hold true.

(a) If $p=2$, then there exists a unique solution $u \in L^{2}\left(0, T ; \mathcal{D}\left((-L)^{\gamma+1}\right)\right)$ to (1.1) (1.3). Moreover, there exists a constant $C>0$ such that

$$
\|u\|_{L^{2}\left(0, T ; \mathcal{D}\left((-L)^{\gamma+1}\right)\right)} \leq C\|F\|_{L^{2}\left(0, T ; \mathcal{D}\left((-L)^{\gamma}\right)\right)} .
$$

(b) If $p \neq 2$, then there exists a unique solution $u \in L^{p}\left(0, T ; \mathcal{D}\left((-L)^{\gamma+1-\tau}\right)\right)$ to (1.1)-(1.3) for any $\tau \in(0,1]$. Moreover, there exists a constant $C>0$ such that

$$
\|u\|_{L^{p}\left(0, T ; \mathcal{D}\left((-L)^{\gamma+1-\tau}\right)\right)} \leq \frac{C}{\tau}\|F\|_{L^{p}\left(0, T ; \mathcal{D}\left((-L)^{\gamma}\right)\right)} .
$$

(c) If $\alpha_{1} p>1$, then for any $\tau \in\left(\frac{1}{\alpha_{1} p}, 1\right]$, there holds

$$
\lim _{t \rightarrow 0}\|u(\cdot, t)\|_{\mathcal{D}\left((-L)^{\gamma+1-\tau}\right)}=0 .
$$

Remark 2.1 We compare the conclusions in Theorems 2.12.2 with those of single-term cases obtained in [26]. In case of the homogeneous source term, i.e. $F=0$ in (1.1), it turns out that Theorem 2.1 is a parallel extension of its single-term counterpart. For instance, in Theorem 
2.1 the regularity results for initial values $a \in L^{2}(\Omega), a \in H_{0}^{1}(\Omega)$ and $a \in H^{2}(\Omega) \cap H_{0}^{1}(\Omega)$ agree with those in [26, Theorem 2.1]. Especially, it will be readily seen from the proof of Theorem 2.1 that the regularity of the solution $u$ at any positive time can be improved from the initial regularity by 2 orders in space, namely, $u(\cdot, t) \in \mathcal{D}\left((-L)^{\gamma+1}\right)$ if $a \in \mathcal{D}\left((-L)^{\gamma}\right)$ for $0<t \leq T$.

On the other hand, if the source term $F$ does not vanish, the improvement of regularity in space is strictly less than 2 orders except for the special case that $F$ is $L^{2}$ in time. For example, if $F \in L^{2}(\Omega \times(0, T))$, then it follows from Theorem 2.2(a) that $u \in L^{2}\left(0, T ; H^{2}(\Omega) \cap H_{0}^{1}(\Omega)\right)$, which coincides with [26, Theorem 2.2]. However, if $F \in L^{p}\left(0, T ; L^{2}(\Omega)\right)$ with $p \neq 2$, then Theorem 2.2(b) asserts $u \in L^{p}\left(0, T ; \mathcal{D}\left((-L)^{1-\tau}\right)\right)$, where $\tau \in(0,1]$ can be arbitrarily small but is never zero. The technical reason is that only in case of $p=2$ one can take advantage of a newly established property in Bazhlekova [3] (see Lemma 3.4).

On basis of these established results, we can consider the dependency of the solution upon some specified coefficients, especially the orders of Caputo derivatives. More precisely, we evaluate the difference between the solutions $u$ and $\widetilde{u}$ to

$$
\begin{cases}\sum_{j=1}^{m} q_{j} \partial_{t}^{\alpha_{j}} u=L_{D} u & \text { in } \Omega \times(0, T] \\ u=0 & \text { on } \partial \Omega \times(0, T] \\ \left.u\right|_{t=0}=a & \text { in } \Omega\end{cases}
$$

and

$$
\begin{cases}\sum_{j=1}^{m} \widetilde{q}_{j} \partial_{t}^{\widetilde{\alpha}_{j}} \widetilde{u}=L_{\widetilde{D}} \widetilde{u} & \text { in } \Omega \times(0, T], \\ \widetilde{u}=0 & \text { on } \partial \Omega \times(0, T], \\ \left.\widetilde{u}\right|_{t=0}=a & \text { in } \Omega\end{cases}
$$

respectively, where $L_{D} u(x, t):=\operatorname{div}(D(x) \nabla u(x, t))$ and $D$ denotes the diffusion coefficient. To this end, we fix $1>\bar{\alpha}>\underline{\alpha}>0, \bar{q}>\underline{q}>0, \delta>0, M>0$ and restrict the coefficients in the admissible sets

$$
\begin{aligned}
& \mathcal{A}:=\left\{\left(\alpha_{1}, \ldots, \alpha_{m}\right) \in \mathbb{R}^{m} ; \bar{\alpha} \geq \alpha_{1}>\alpha_{2}>\cdots>\alpha_{m} \geq \underline{\alpha}\right\}, \\
& \mathcal{Q}:=\left\{\left(q_{1}, \ldots, q_{m}\right) \in \mathbb{R}^{m} ; q_{1}=1, q_{j} \in[\underline{q}, \bar{q}](j=2, \ldots, m)\right\}, \\
& \mathcal{U}:=\left\{D \in C^{1}(\bar{\Omega}) ; D \geq \delta \text { in } \bar{\Omega},\|D\|_{C^{1}(\bar{\Omega})} \leq M\right\} .
\end{aligned}
$$

Under these settings, we can show the following result on the Lipschitz stability.

Theorem 2.3 Fix $\gamma, \tau \in(0,1]$. Let $u$ and $\widetilde{u}$ be the solutions to (2.9) and (2.10) respectively, where

$$
a \in \mathcal{D}\left((-L)^{\gamma}\right), \quad\left(\alpha_{1}, \ldots, \alpha_{m}\right),\left(\widetilde{\alpha}_{1}, \ldots, \widetilde{\alpha}_{m}\right) \in \mathcal{A}, \quad\left(q_{1}, \ldots, q_{m}\right),\left(\widetilde{q}_{1}, \ldots, \widetilde{q}_{m}\right) \in \mathcal{Q}, \quad D, \widetilde{D} \in \mathcal{U}
$$

and $\mathcal{A}, \mathcal{Q}, \mathcal{U}$ are defined in (2.11). Then there exists a constant $C>0$ depending only on $a, \mathcal{A}, \mathcal{Q}$ and $\mathcal{U}$ such that

$$
\|u-\widetilde{u}\|_{L^{\frac{1}{1-\gamma}}\left(0, T ; \mathcal{D}\left((-L)^{1-\tau}\right)\right)} \leq \frac{C}{\tau}\left(\sum_{j=1}^{m}\left|\alpha_{j}-\widetilde{\alpha}_{j}\right|+\sum_{j=2}^{m}\left|q_{j}-\widetilde{q}_{j}\right|+\|D-\widetilde{D}\|_{C^{1}(\bar{\Omega})}\right)
$$


for $0<\gamma<\frac{1}{2}$ and

$$
\|u-\widetilde{u}\|_{L^{2}\left(0, T ; H^{2}(\Omega)\right)} \leq C\left(\sum_{j=1}^{m}\left|\alpha_{j}-\widetilde{\alpha}_{j}\right|+\sum_{j=2}^{m}\left|q_{j}-\widetilde{q}_{j}\right|+\|D-\widetilde{D}\|_{C^{1}(\bar{\Omega})}\right)
$$

for $\gamma \geq \frac{1}{2}$.

The above theorem extends a similar result in $[13$ for the single-term case. It is also fundamental for the optimization method for an inverse problem of determining $\alpha_{j}, q_{j}, D(x)$ by extra data of the solution.

In Sakamoto \& Yamamoto [26, the decay rate of the solution to the single-term timefractional diffusion equation (1.4) was shown to be $t^{-\alpha}$ as $t \rightarrow \infty$. Here we give generalization for the multi-term case where we specify the principal term of the solution as $t \rightarrow \infty$.

Theorem 2.4 Let $F=0$ and $a \in L^{2}(\Omega)$. Then there exists a unique solution $u \in$ $C\left([0, \infty) ; L^{2}(\Omega)\right) \cap C\left((0, \infty) ; H^{2}(\Omega) \cap H_{0}^{1}(\Omega)\right)$ to (1.1)-(1.3). Moreover, there exists a constant $C>0$ such that

$$
\left\|u(\cdot, t)-\frac{(-L)^{-1}\left(q_{m} a\right)}{\Gamma\left(1-\alpha_{m}\right) t^{\alpha_{m}}}\right\|_{H^{2}(\Omega)} \leq \frac{C\|a\|_{L^{2}(\Omega)}}{t^{\alpha_{m-1}}} \text { as } t \rightarrow \infty .
$$

Remark 2.2 We explain the significance of the Theorem 2.4 It reveals that the decay rate of $u(\cdot, t)$ in sense of $H^{2}(\Omega)$ is exactly $t^{-\alpha_{m}}$ as $t \rightarrow \infty$. In fact, inequality (2.14) implies that there exist constants $C_{2}>C_{1}>0$ such that

$$
C_{1}\|a\|_{L^{2}(\Omega)} t^{-\alpha_{m}} \leq\|u(\cdot, t)\|_{H^{2}(\Omega)} \leq C_{2}\|a\|_{L^{2}(\Omega)} t^{-\alpha_{m}} \quad \text { as } t \rightarrow \infty .
$$

Consequently, it turns out that the decay rate $t^{-\alpha_{m}}$ is the best possible. In other words, if

$$
\|u(\cdot, t)\|_{H^{2}(\Omega)} \leq C t^{-\beta} \text { as } t \rightarrow \infty
$$

for any order $\beta>\alpha_{m}$ and some constant $C>0$, then $u(x, t)=0$ for $x \in \Omega$ and $t>0$. Actually, in this case it is easily inferred from the lower bound in (2.15) that there should be $a=0$ in $\Omega$. Therefore, Theorem 2.1 and the upper bound in (2.15) immediately imply $u \equiv 0$ in $\Omega \times(0, \infty)$. Furthermore, (2.14) also gives the convergence rate of the approximation

$$
u(\cdot, t)-\frac{(-L)^{-1}\left(q_{m} a\right)}{\Gamma\left(1-\alpha_{m}\right) t^{-\alpha_{m}}} \rightarrow 0 \text { in } H^{2}(\Omega) \text { as } t \rightarrow \infty,
$$

that is, $t^{-\alpha_{m-1}}$.

\section{Proofs of Main Results}

In this section, we give proofs for the theorems stated in Section 2 ,

In the discussion of single-term time-fractional diffusion equations, it turns out that the solutions can be explicitly represented by the usual Mittag-Leffler function

$$
E_{\alpha, \beta}(z):=\sum_{k=0}^{\infty} \frac{z^{k}}{\Gamma(\alpha k+\beta)}, \quad z \in \mathbb{C}, \alpha>0, \beta \in \mathbb{R},
$$


and several basic properties play remarkable roles especially for obtaining estimates for the stability. Since explicit solutions to the multi-term case are also available by using a generalized form of (3.1) called the multinomial Mittag-Leffler function, we shall first investigate this generalization so that similar arguments are still feasible for multi-term time-fractional diffusion equations.

\subsection{Properties of multinomial Mittag-Leffler functions}

The multinomial Mittag-Leffler function is defined as (see Luchko \& Gorenflo [19])

$$
E_{\left(\beta_{1}, \ldots, \beta_{m}\right), \beta_{0}}\left(z_{1}, \ldots, z_{m}\right):=\sum_{k=0}^{\infty} \sum_{k_{1}+\cdots+k_{m}=k} \frac{\left(k ; k_{1}, \ldots, k_{m}\right) \prod_{j=1}^{m} z_{j}^{k_{j}}}{\Gamma\left(\beta_{0}+\sum_{j=1}^{m} \beta_{j} k_{j}\right)},
$$

where we assume $0<\beta_{0}<2,0<\beta_{j}<1, z_{j} \in \mathbb{C}(j=1, \ldots, m)$, and $\left(k ; k_{1}, \ldots, k_{m}\right)$ denotes the multinomial coefficient

$$
\left(k ; k_{1}, \ldots, k_{m}\right):=\frac{k !}{k_{1} ! \cdots k_{m} !} \quad \text { with } k=\sum_{j=1}^{m} k_{j},
$$

where $k_{j}, 1 \leq j \leq m$, are non-negative integers. We recall the following formula for multinomial coefficients (see Berge [5])

$$
\sum_{j=1}^{m}\left(k-1 ; k_{1}, \ldots, k_{j-1}, k_{j}-1, k_{j+1}, \ldots, k_{m}\right)=\left(k ; k_{1}, \ldots, k_{m}\right) .
$$

If some $k_{j_{0}}$ vanishes, we understand $\left(k-1 ; k_{1}, \ldots, k_{j_{0}-1}, k_{j_{0}}-1, k_{j_{0}+1}, \ldots, k_{m}\right)=0$ and (3.3) degenerates to its lower dimensional version.

Concerning the relation between multinomial Mittag-Leffler functions with different parameters, we have the following lemma.

Lemma 3.1 Let $0<\beta_{0}<2,0<\beta_{j}<1(j=1, \ldots, m)$ and $z_{j} \in \mathbb{C}(j=1, \ldots, m)$ be fixed. Then

$$
\frac{1}{\Gamma\left(\beta_{0}\right)}+\sum_{j=1}^{m} z_{j} E_{\left(\beta_{1}, \ldots, \beta_{m}\right), \beta_{0}+\beta_{j}}\left(z_{1}, \ldots, z_{m}\right)=E_{\left(\beta_{1}, \ldots, \beta_{m}\right), \beta_{0}}\left(z_{1}, \ldots, z_{m}\right) .
$$

Proof. According to definition (3.2), direct calculations yield

$$
\begin{aligned}
& \sum_{j=1}^{m} z_{j} E_{\left(\beta_{1}, \ldots, \beta_{m}\right), \beta_{0}+\beta_{j}}\left(z_{1}, \ldots, z_{m}\right) \\
= & \sum_{j=1}^{m} \sum_{k=0}^{\infty} \sum_{k_{1}+\cdots+k_{m}=k} \frac{\left(k ; k_{1}, \ldots, k_{m}\right) z_{j} \prod_{\ell=1}^{m} z_{\ell}^{k_{\ell}}}{\Gamma\left(\beta_{0}+\beta_{j}+\sum_{\ell=1}^{m} \beta_{\ell} k_{\ell}\right)} \\
= & \sum_{k=0}^{\infty} \sum_{j=1}^{m}\left\{\frac{z_{j}^{k+1}}{\Gamma\left(\beta_{0}+\beta_{j}(k+1)\right)}+\sum_{\substack{k_{1}+\cdots+k_{m}=k \\
k_{j}<k}} \frac{\left(k ; k_{1}, \ldots, k_{m}\right) z_{j} \prod_{\ell=1}^{m} z_{\ell}^{k_{\ell}}}{\Gamma\left(\beta_{0}+\beta_{j}+\sum_{\ell=1}^{m} \beta_{\ell} k_{\ell}\right)}\right\} \\
= & \sum_{k=0}^{\infty} \sum_{j=1}^{m}\left\{\frac{z_{j}^{k+1}}{\Gamma\left(\beta_{0}+\beta_{j}(k+1)\right)}+\sum_{\substack{k_{1}+\cdots+k_{m}=k+1 \\
0<k_{j}<k+1}} \frac{\left(k ; k_{1}, \ldots, k_{j-1}, k_{j}-1, k_{j+1}, \ldots, k_{m}\right) \prod_{\ell=1}^{m} z_{\ell}^{k_{\ell}}}{\Gamma\left(\beta_{0}+\sum_{\ell=1}^{m} \beta_{\ell} k_{\ell}\right)}\right\}
\end{aligned}
$$




$$
\begin{aligned}
= & \sum_{k=0}^{\infty}\left\{\sum_{j=1}^{m} \frac{z_{j}^{k+1}}{\Gamma\left(\beta_{0}+\beta_{j}(k+1)\right)}+\sum_{\substack{k_{1}+\cdots+k_{m}=k+1 \\
k_{\ell}<k+1(\forall \ell)}} \frac{\left(k+1 ; k_{1}, \ldots, k_{m}\right) \prod_{\ell=1}^{m} z_{\ell}^{k_{\ell}}}{\Gamma\left(\beta_{0}+\sum_{\ell=1}^{m} \beta_{\ell} k_{\ell}\right)}\right\} \\
= & \sum_{k=0}^{\infty} \sum_{k_{1}+\cdots+k_{m}=k+1} \frac{\left(k+1 ; k_{1}, \ldots, k_{m}\right) \prod_{\ell=1}^{m} z_{j}^{k_{j}}}{\Gamma\left(\beta_{0}+\sum_{\ell=1}^{m} \beta_{\ell} k_{\ell}\right)} \\
= & \sum_{k=1}^{\infty} \sum_{k_{1}+\cdots+k_{m}=k} \frac{\left(k ; k_{1}, \ldots, k_{m}\right) \prod_{\ell=1}^{m} z_{\ell}^{k_{\ell}}}{\Gamma\left(\beta_{0}+\sum_{\ell=1}^{m} \beta_{\ell} k_{\ell}\right)}=E_{\left(\beta_{1}, \ldots, \beta_{m}\right), \beta_{0}}\left(z_{1}, \ldots, z_{m}\right)-\frac{1}{\Gamma\left(\beta_{0}\right)},
\end{aligned}
$$

where we apply formula (3.3) to obtain (3.5). In (3.4), we distill the case $k_{j}=k$ in the $j$-th term and substitute $k_{j}+1$ with $k_{j}$ for the others to proceed to the next equality.

Concerning the regularity of the solution to a single-term time-fractional diffusion equation, the estimate (see [24, p. 35])

$$
\left|E_{\alpha, \beta}(-\eta)\right| \leq \frac{C}{1+\eta}, \quad \eta \geq 0
$$

is essential. Here we extend the above inequality to the multinomial case by a complex variable argument.

Lemma 3.2 Let $0<\beta<2$ and $1>\alpha_{1}>\cdots>\alpha_{m}>0$ be given. Assume that $\alpha_{1} \pi / 2<$ $\mu<\alpha_{1} \pi, \mu \leq\left|\arg \left(z_{1}\right)\right| \leq \pi$ and there exists $K>0$ such that $-K \leq z_{j}<0(j=2, \ldots, m)$. Then there exists a constant $C>0$ depending only on $\mu, K, \alpha_{j}(j=1, \ldots, m)$ and $\beta$ such that

$$
\left|E_{\left(\alpha_{1}, \alpha_{1}-\alpha_{2}, \ldots, \alpha_{1}-\alpha_{m}\right), \beta}\left(z_{1}, \ldots, z_{m}\right)\right| \leq \frac{C}{1+\left|z_{1}\right|} .
$$

Proof. Let $\alpha_{j}, z_{j}(j=1, \ldots, m)$ and $\beta$ be assumed as above and introduce the notation

$$
E_{\boldsymbol{\alpha}^{\prime}, \beta}\left(z_{1}, \ldots, z_{m}\right):=E_{\left(\alpha_{1}, \alpha_{1}-\alpha_{2}, \ldots, \alpha_{1}-\alpha_{m}\right), \beta}\left(z_{1}, \ldots, z_{m}\right) .
$$

In the sequel, we denote by $C$ a general positive constant depending at most on $\mu, K, \alpha_{j}$ $(j=1, \ldots, m)$ and $\beta$. First we rewrite the multinomial Mittag-Leffler function (3.2) in an alternative form with the aid of the contour integral representation of $1 / \Gamma(z)$ (see [24, §1.1.6]) that

$$
\frac{1}{\Gamma(z)}=\frac{1}{2 \alpha_{1} \pi \mathrm{i}} \int_{\gamma(R, \theta)} \exp \left(\zeta^{1 / \alpha_{1}}\right) \zeta^{\left(1-z-\alpha_{1}\right) / \alpha_{1}} \mathrm{~d} \zeta
$$

where $R>0$ is a constant to be determined later and $\alpha_{1} \pi / 2<\theta<\mu$. Here $\gamma(R, \theta)$ denotes the contour

$$
\gamma(R, \theta):=\{\zeta \in \mathbb{C} ;|\zeta|=R,|\arg (\zeta)| \leq \theta\} \cup\{\zeta \in \mathbb{C} ;|\zeta|>R,|\arg (\zeta)|= \pm \theta\} .
$$

Then it follows from the multinomial formula that

$$
\begin{aligned}
& E_{\boldsymbol{\alpha}^{\prime}, \beta}\left(z_{1}, \ldots, z_{m}\right) \\
= & \frac{1}{2 \alpha_{1} \pi \mathrm{i}} \sum_{k=0}^{\infty} \sum_{k_{1}+\cdots+k_{m}=k}\left(k ; k_{1}, \ldots, k_{m}\right) \prod_{j=1}^{m} z_{j}^{k_{j}}
\end{aligned}
$$




$$
\begin{array}{r}
\times\left\{\int_{\gamma(R, \theta)} \exp \left(\zeta^{1 / \alpha_{1}}\right) \zeta^{\left(1-\beta-\alpha_{1}(k+1)-\alpha_{2} k_{2}-\cdots-\alpha_{m} k_{m}\right) / \alpha_{1}} \mathrm{~d} \zeta\right\} \\
=\frac{1}{2 \alpha_{1} \pi \mathrm{i}} \int_{\gamma(R, \theta)} \exp \left(\zeta^{1 / \alpha_{1}}\right) \zeta^{(1-\beta) / \alpha_{1}-1} \\
\times \sum_{k=0}^{\infty}\left\{\sum_{k_{1}+\cdots+k_{m}=k}\left(k ; k_{1}, \ldots, k_{m}\right)\left(\frac{z_{1}}{\zeta}\right)^{k_{1}} \prod_{j=2}^{m}\left(\frac{z_{j}}{\zeta^{1-\alpha_{j} / \alpha_{1}}}\right)^{k_{j}}\right\} \mathrm{d} \zeta \\
=\frac{1}{2 \alpha_{1} \pi \mathrm{i}} \int_{\gamma(R, \theta)} \exp \left(\zeta^{1 / \alpha_{1}}\right) \zeta^{(1-\beta) / \alpha_{1}-1} \sum_{k=0}^{\infty}\left(\frac{z_{1}}{\zeta}+\sum_{j=2}^{m} \frac{z_{k}}{\zeta^{1-\alpha_{j} / \alpha_{1}}}\right)^{k} \mathrm{~d} \zeta .
\end{array}
$$

In order to guarantee the convergence of the summation with respect to $k$, it is required that

$$
\left|\frac{z_{1}}{\zeta}+\sum_{j=2}^{m} \frac{z_{j}}{\zeta^{1-\alpha_{j} / \alpha_{1}}}\right|<1, \quad \forall \zeta \in \gamma(R, \theta) .
$$

Since $\left|z_{j}\right| \leq K$ for $j=2, \ldots, m$, the above inequality is achieved by taking $R$ such that

$$
R>\left|z_{1}\right|+K \sum_{j=2}^{m} R^{\alpha_{j} / \alpha_{1}}
$$

Moreover, if we restrict, for example, $\left|z_{1}\right| \leq K$, then $R$ can be fixed as a constant depending only on $K$ and $\alpha_{j}(j=1, \ldots, m)$. Now we deduce for $\left|z_{j}\right| \leq K(j=1, \ldots, m)$ that

$$
E_{\boldsymbol{\alpha}^{\prime}, \beta}\left(z_{1}, \ldots, z_{m}\right)=\frac{1}{2 \alpha_{1} \pi \mathrm{i}} \int_{\gamma(R, \theta)} \frac{\exp \left(\zeta^{1 / \alpha_{1}}\right) \zeta^{(1-\beta) / \alpha_{1}}}{\zeta-z_{1}-\sum_{j=2}^{m} z_{j} \zeta^{\alpha_{j} / \alpha_{1}}} \mathrm{~d} \zeta .
$$

Next we fix $z_{2}, \ldots, z_{m}$ as negative parameters and regard both sides of (3.6) as functions of the single complex variable $z_{1}$, which allows the application of the principle of analytic continuation to extend equality (3.6) to a domain including $\left\{z_{1} \in \mathbb{C} ; \mu \leq\left|\arg \left(z_{1}\right)\right| \leq \pi\right\}$ (see Figure (1).

For $\left|z_{1}\right|>R$, we investigate the denominator of the integrand in (3.6). Since $z_{j}<0$ and $\alpha_{j}<\alpha_{1}$ for $j=2, \ldots, m$, it turns out that the curve $\zeta-\sum_{j=2}^{m} z_{j} \zeta^{\alpha_{j} / \alpha_{1}}(\zeta \in \gamma(R, \theta))$ locates on the right-hand side of $\gamma(R, \theta)$; that is, $\gamma(R, \theta)$ is shifted by the term $-\sum_{j=2}^{m} z_{j} \zeta^{\alpha_{j} / \alpha_{1}}$ to the positive direction. This observation immediately implies

$$
\min _{\zeta \in \gamma(R, \theta)}\left|\zeta-z_{1}-\sum_{j=2}^{m} z_{j} \zeta^{\alpha_{j} / \alpha_{1}}\right| \geq \min _{\zeta \in \gamma(R, \theta)}\left|\zeta-z_{1}\right| \geq\left|z_{1}\right| \sin (\mu-\theta) .
$$

Therefore, we come up with the estimate

$$
\begin{aligned}
\left|E_{\boldsymbol{\alpha}^{\prime}, \beta}\left(z_{1}, \ldots, z_{m}\right)\right| & =\frac{1}{2 \alpha_{1} \pi}\left|\int_{\gamma(R, \theta)} \frac{\exp \left(\zeta^{1 / \alpha_{1}}\right) \zeta^{(1-\beta) / \alpha_{1}}}{\zeta-z_{1}-\sum_{j=2}^{m} z_{j} \zeta^{\alpha_{j} / \alpha_{1}}} \mathrm{~d} \zeta\right| \\
& \leq\left(\frac{1}{2 \alpha_{1} \pi \sin (\mu-\theta)} \int_{\gamma(R, \theta)}\left|\exp \left(\zeta^{1 / \alpha_{1}}\right)\right|\left|\zeta^{(1-\beta) / \alpha_{1}}\right| \mathrm{d} \zeta\right) \frac{1}{\left|z_{1}\right|}
\end{aligned}
$$




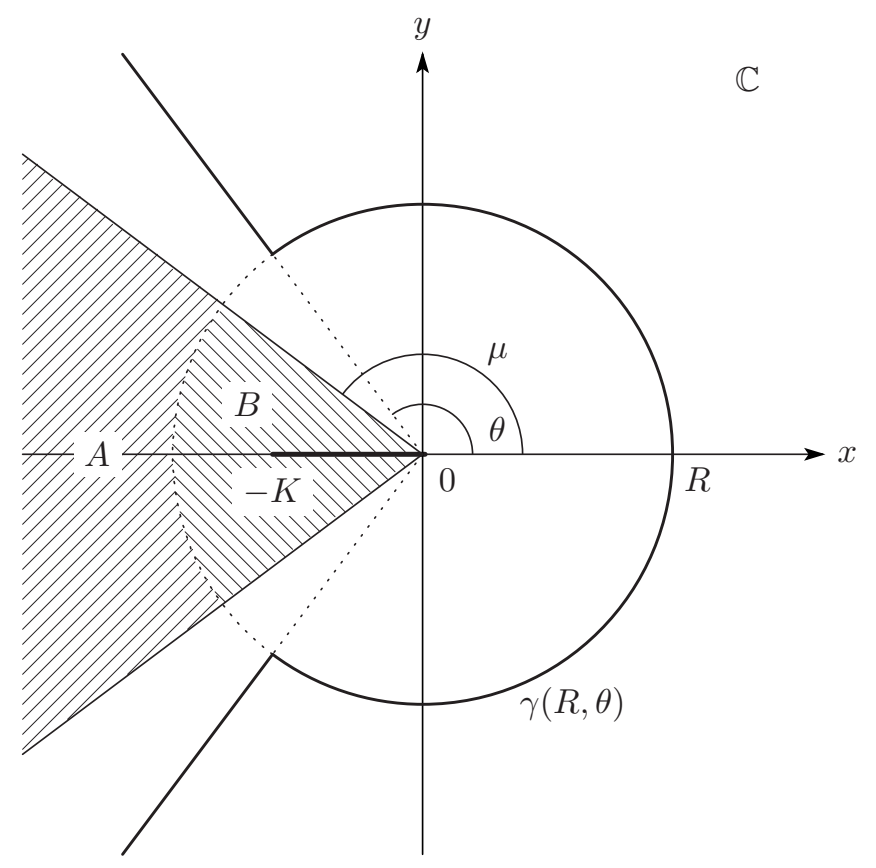

Figure 1. Settings of Lemma 3.2 and the contour $\gamma(R, \theta)$. If $z_{1}$ is located in the shaded domain $A$, we employ the principle of analytic continuation and the contour integral representation

(3.6). When $z_{1}$ is in the shaded domain $B$, it suffices to argue by definition (3.2).

The integral along $\gamma(R, \theta)$ converges, because for $\zeta$ such that $\arg (\zeta)= \pm \theta$ and $|\zeta|>R$, there holds

$$
\left|\exp \left(\zeta^{1 / \alpha_{1}}\right)\right|=\exp \left(|\zeta|^{1 / \alpha_{1}} \cos \left(\theta / \alpha_{1}\right)\right) \quad \text { with } \cos \left(\theta / \alpha_{1}\right)<0,
$$

while the integral on the $\operatorname{arc}\{\zeta \in \mathbb{C} ;|\zeta|=R,|\arg (\zeta)| \leq \theta\}$ is a constant. Consequently

$$
\left|E_{\boldsymbol{\alpha}^{\prime}, \beta}\left(z_{1}, \ldots, z_{m}\right)\right| \leq \frac{C}{\left|z_{1}\right|}, \quad \mu \leq\left|\arg \left(z_{1}\right)\right| \leq \pi,\left|z_{1}\right|>R .
$$

For $\mu \leq\left|\arg \left(z_{1}\right)\right| \leq \pi$ such that $\left|z_{1}\right| \leq R$, it is directly verified that

$$
\begin{aligned}
\left|E_{\boldsymbol{\alpha}^{\prime}, \beta}\left(z_{1}, \ldots, z_{m}\right)\right| & =\left|\sum_{k=0}^{\infty} \sum_{k_{1}+\cdots+k_{m}=k} \frac{\left(k ; k_{1}, \ldots, k_{m}\right) \prod_{j=1}^{m} z_{j}^{k_{j}}}{\Gamma\left(\beta+\alpha_{1} k-\sum_{j=2}^{m} \alpha_{j} k_{j}\right)}\right| \\
& \leq \sum_{k=0}^{\infty} \sum_{k_{1}+\cdots+k_{m}=k} \frac{\left(k ; k_{1}, \ldots, k_{m}\right) \prod_{j=1}^{m}\left|z_{j}\right|^{k_{j}}}{\Gamma\left(\beta+\alpha_{1} k-\sum_{j=2}^{m} \alpha_{j} k_{j}\right)} \\
& \leq C \sum_{k=0}^{\infty} \sum_{k_{1}+\cdots+k_{m}=k} \frac{\left(k ; k_{1}, \ldots, k_{m}\right) \prod_{j=1}^{m}\left|z_{j}\right|^{k_{j}}}{\Gamma\left(\beta+\left(\alpha_{1}-\alpha_{2}\right) k\right)} \\
& =C \sum_{k=0}^{\infty} \frac{1}{\Gamma\left(\beta+\left(\alpha_{1}-\alpha_{2}\right) k\right)}\left(\sum_{j=1}^{m}\left|z_{j}\right|\right)^{k} \leq C \sum_{k=0}^{\infty} \frac{(R+(m-1) K)^{k}}{\Gamma\left(\beta+\left(\alpha_{1}-\alpha_{2}\right) k\right)} \leq C,
\end{aligned}
$$

which, together with (3.7), finishes the proof. 
For later use, we adopt the abbreviation

$$
E_{\boldsymbol{\alpha}^{\prime}, \beta}^{(n)}(t):=E_{\left(\alpha_{1}, \alpha_{1}-\alpha_{2}, \ldots, \alpha_{1}-\alpha_{m}\right), \beta}\left(-\lambda_{n} t^{\alpha_{1}},-q_{2} t^{\alpha_{1}-\alpha_{2}}, \ldots,-q_{m} t^{\alpha_{1}-\alpha_{m}}\right), \quad t>0,
$$

where $\lambda_{n}$ is the $n$-th eigenvalue of $-L, 0<\beta<2$, and $\alpha_{j}, q_{j}$ are those positive constants in (1.1). Especially, regarding the derivative of $t^{\alpha_{1}} E_{\boldsymbol{\alpha}^{\prime}, 1+\alpha_{1}}^{(n)}(t)$ with respect to $t>0$, we state the following technical lemma.

Lemma 3.3 Let $1>\alpha_{1}>\cdots>\alpha_{m}>1$. Then

$$
\frac{\mathrm{d}}{\mathrm{d} t}\left\{t^{\alpha_{1}} E_{\boldsymbol{\alpha}^{\prime}, 1+\alpha_{1}}^{(n)}(t)\right\}=t^{\alpha_{1}-1} E_{\boldsymbol{\alpha}^{\prime}, \alpha_{1}}^{(n)}(t), \quad t>0 .
$$

Proof. By definition, we carry out a direct differentiation and utilize the formula $\Gamma(s)=\Gamma(s+$ $1) / s$ to derive

$$
\begin{aligned}
& \frac{\mathrm{d}}{\mathrm{d} t}\left\{t^{\alpha_{1}} E_{\boldsymbol{\alpha}^{\prime}, 1+\alpha_{1}}^{(n)}(t)\right\} \\
= & \frac{\mathrm{d}}{\mathrm{d} t}\left\{\sum_{k=0}^{\infty} \sum_{k_{1}+\cdots+k_{m}=k} \frac{\left(k ; k_{1}, \ldots, k_{m}\right)\left(-\lambda_{n}\right)^{k_{1}} \prod_{j=2}^{m}\left(-q_{j}\right)^{k_{j}} t^{\alpha_{1}(k+1)-\alpha_{2} k_{2}-\cdots-\alpha_{m} k_{m}}}{\Gamma\left(1+\alpha_{1}(k+1)-\sum_{j=2}^{m} \alpha_{j} k_{j}\right)}\right\} \\
= & \sum_{k=0}^{\infty} \sum_{k_{1}+\cdots+k_{m}=k} \frac{\left(k ; k_{1}, \ldots, k_{m}\right)\left(-\lambda_{n}\right)^{k_{1}} \prod_{j=2}^{m}\left(-q_{j}\right)^{k_{j}} t^{\alpha_{1}(k+1)-\alpha_{2} k_{2}-\cdots-\alpha_{m} k_{m}-1}}{\Gamma\left(\alpha_{1}(k+1)-\sum_{j=2}^{m} \alpha_{j} k_{j}\right)} \\
= & t^{\alpha_{1}-1} \sum_{k=0}^{\infty} \sum_{k_{1}+\cdots+k_{m}=k} \frac{\left(k ; k_{1}, \ldots, k_{m}\right)\left(-\lambda_{n} t^{\alpha_{1}}\right)^{k_{1}} \prod_{j=2}^{m}\left(-q_{j} t^{\alpha_{1}-\alpha_{j}}\right)^{k_{j}}}{\Gamma\left(\alpha_{1}+\alpha_{1} k_{1}+\sum_{j=2}^{m}\left(\alpha_{1}-\alpha_{j}\right) k_{j}\right)} \\
= & t^{\alpha_{1}-1} E_{\boldsymbol{\alpha}^{\prime}, \alpha_{1}}^{(n)}(t) .
\end{aligned}
$$

Here we use the fact that $t^{\alpha_{1}} E_{\boldsymbol{\alpha}^{\prime}, 1+\alpha_{1}}^{(n)}(t)$ is real analytic for $t>0$ so that termwise differentiations are available.

\subsection{Proofs of Theorems 2.12 .3}

Now we are ready to employ the multinomial Mittag-Leffler functions to show results on the well-posedness. For later use we recall the eigensystem $\left\{\lambda_{n}, \varphi_{n}\right\}$ of the elliptic operator $-L$ and the abbreviation $E_{\boldsymbol{\alpha}^{\prime}, \beta}^{(n)}(t)(0<\beta<2)$ in (3.8).

First we prove Theorem 2.1, that is, the case of vanishing source term $F$. It was shown in 18 that the explicit solution to (1.1)-(1.3) is given by

$$
u(\cdot, t)=\sum_{n=1}^{\infty}\left(1-\lambda_{n} t^{\alpha_{1}} E_{\boldsymbol{\alpha}^{\prime}, 1+\alpha_{1}}^{(n)}(t)\right)\left(a, \varphi_{n}\right) \varphi_{n} .
$$

With the aid of Lemmata 3.13 .3 it is straightforward to demonstrate the well-posedness by dominating the solution by the initial value.

Proof of Theorem 2.1. Let $a \in \mathcal{D}\left((-L)^{\gamma}\right)$ with $0 \leq \gamma \leq 1$. In the sequel, by $C$ we refer to positive constants independent of the initial value $a$ which may vary from line by line.

(a) First, a direct application of Lemma 3.2 yields

$$
\left|1-\lambda_{n} t^{\alpha_{1}} E_{\boldsymbol{\alpha}^{\prime}, 1+\alpha_{1}}^{(n)}(t)\right| \leq 1+\lambda_{n} t^{\alpha_{1}} \frac{C}{1+\lambda_{n} t^{\alpha_{1}}} \leq C .
$$


Thus, we take advantage of (3.9) to derive

$$
\|u(\cdot, t)\|_{L^{2}(\Omega)}=\left\{\sum_{n=1}^{\infty}\left|1-\lambda_{n} t^{\alpha_{1}} E_{\boldsymbol{\alpha}^{\prime}, 1+\alpha_{1}}^{(n)}(t)\right|^{2}\left|\left(a, \varphi_{n}\right)\right|^{2}\right\}^{1 / 2} \leq C\|a\|_{L^{2}(\Omega)}
$$

for $0<t \leq T$, where we use the fact that $\left\{\varphi_{n}\right\}$ forms an orthonormal basis of $L^{2}(\Omega)$. Since the summation in (3.9) converges in $L^{2}(\Omega)$ uniformly in $t \in[0, T]$, we get $u \in C\left([0, T] ; L^{2}(\Omega)\right)$ or (2.1). Furthermore, by the definition of $\mathcal{D}(-L)$, we see

$$
\|u(\cdot, t)\|_{\mathcal{D}(-L)}^{2}=\sum_{n=1}^{\infty}\left(\lambda_{n}\left|1-\lambda_{n} t^{\alpha_{1}} E_{\boldsymbol{\alpha}^{\prime}, 1+\alpha_{1}}^{(n)}(t)\right|\right)^{2}\left|\left(a, \varphi_{n}\right)\right|^{2}
$$

In order to treat the term $1-\lambda_{n} t^{\alpha_{1}} E_{\boldsymbol{\alpha}^{\prime}, 1+\alpha_{1}}^{(n)}(t)$, we substitute

$$
\beta_{0}=1, \quad \beta_{1}=\alpha_{1}, \quad z_{1}=-\lambda_{n} t^{\alpha_{1}}, \quad \beta_{j}=\alpha_{1}-\alpha_{j} \text { and } z_{j}=-q_{j} t^{\alpha_{1}-\alpha_{j}}(j=2, \ldots, m)
$$

in Lemma 3.1 and then utilize Lemma 3.2 to deduce

$$
\begin{aligned}
\left|1-\lambda_{n} t^{\alpha_{1}} E_{\boldsymbol{\alpha}^{\prime}, 1+\alpha_{1}}^{(n)}(t)\right| & =\left|E_{\boldsymbol{\alpha}^{\prime}, 1}^{(n)}(t)+\sum_{j=2}^{m} q_{j} t^{\alpha_{1}-\alpha_{j}} E_{\boldsymbol{\alpha}^{\prime}, 1+\alpha_{1}-\alpha_{j}}^{(n)}(t)\right| \\
& \leq\left|E_{\boldsymbol{\alpha}^{\prime}, 1}^{(n)}(t)\right|+C \sum_{j=2}^{m} t^{\alpha_{1}-\alpha_{j}}\left|E_{\boldsymbol{\alpha}^{\prime}, 1+\alpha_{1}-\alpha_{j}}^{(n)}(t)\right| \leq C \sum_{j=1}^{m} \frac{t^{\alpha_{1}-\alpha_{j}}}{1+\lambda_{n} t^{\alpha_{1}}} .
\end{aligned}
$$

Therefore, for $0<t \leq T$, we estimate

$$
\begin{aligned}
\|u(\cdot, t)\|_{\mathcal{D}(-L)}^{2} & =\sum_{n=1}^{\infty}\left|\lambda_{n}^{1-\gamma}\left(1-\lambda_{n} t^{\alpha_{1}} E_{\boldsymbol{\alpha}^{\prime}, 1+\alpha_{1}}^{(n)}(t)\right)\right|^{2}\left|\lambda_{n}^{\gamma}\left(a, \varphi_{n}\right)\right|^{2} \\
& \leq C^{2} \sum_{n=1}^{\infty}\left(\sum_{j=1}^{m} \frac{\lambda_{n}^{1-\gamma} t^{\alpha_{1}-\alpha_{j}}}{1+\lambda_{n} t^{\alpha_{1}}}\right)^{2}\left|\lambda_{n}^{\gamma}\left(a, \varphi_{n}\right)\right|^{2} \\
& \leq C^{2} \sum_{n=1}^{\infty}\left(\sum_{j=1}^{m} \frac{\left(\lambda_{n} t^{\alpha_{1}}\right)^{1-\gamma}}{1+\lambda_{n} t^{\alpha_{1}}} t^{\alpha_{1} \gamma-\alpha_{j}}\right)^{2}\left|\lambda_{n}^{\gamma}\left(a, \varphi_{n}\right)\right|^{2} \\
& \leq C^{2}\left(\sum_{j=1}^{m} t^{\alpha_{1} \gamma-\alpha_{j}}\right)^{2} \sum_{n=1}^{\infty}\left|\lambda_{n}^{\gamma}\left(a, \varphi_{n}\right)\right|^{2} \leq\left(C\|a\|_{\left.\mathcal{D}((-L))^{\gamma} t^{\alpha_{1}(\gamma-1)}\right)^{2}}\right.
\end{aligned}
$$

where we use the fact

$$
\frac{\left(\lambda_{n} t^{\alpha_{1}}\right)^{1-\gamma}}{1+\lambda_{n} t^{\alpha_{1}}} \leq\left\{\begin{array}{ll}
\frac{1}{1+\lambda_{n} t^{\alpha_{1}}} & \text { if } \lambda_{n} t^{\alpha_{1}} \leq 1 \\
\frac{\lambda_{n} t^{\alpha_{1}}}{1+\lambda_{n} t^{\alpha_{1}}} & \text { if } \lambda_{n} t^{\alpha_{1}} \geq 1
\end{array}\right\} \leq 1
$$

in the third inequality. This, together with the fact $\mathcal{D}(-L) \subset H^{2}(\Omega)$, yield the estimate (2.2). Furthermore, it follows immediately from (2.2) and $\alpha_{1}<1$ that $u \in L^{\frac{1}{1-\gamma}}\left(0, T ; H^{2}(\Omega) \cap H_{0}^{1}(\Omega)\right)$.

(b) In order to investigate the asymptotic behavior near $t=0$, first we have

$$
\|u(\cdot, t)-a\|_{\mathcal{D}\left((-L)^{\gamma}\right)}^{2}=\sum_{n=1}^{\infty}\left|\lambda_{n} t^{\alpha_{1}} E_{\boldsymbol{\alpha}^{\prime}, 1+\alpha_{1}}^{(n)}(t)\right|^{2}\left|\lambda_{n}^{\gamma}\left(a, \varphi_{n}\right)\right|^{2} \leq\left(C\|a\|_{\mathcal{D}\left((-L)^{\gamma}\right)}\right)^{2}<\infty
$$


for $0 \leq t \leq T$ by a direct calculation and Lemma 3.2. On the other hand, in view of Lemma 3.1 the term $\lambda_{n} t^{\alpha_{1}} E_{\boldsymbol{\alpha}^{\prime}, 1+\alpha_{1}}^{(n)}(t)$ can be rewritten as

$$
\lambda_{n} t^{\alpha_{1}} E_{\boldsymbol{\alpha}^{\prime}, 1+\alpha_{1}}^{(n)}(t)=-\left(E_{\boldsymbol{\alpha}^{\prime}, 1}^{(n)}(t)-1\right)-\sum_{j=2}^{m} q_{j} t^{\alpha_{1}-\alpha_{j}} E_{\boldsymbol{\alpha}^{\prime}, 1+\alpha_{1}-\alpha_{j}}^{(n)}(t) .
$$

Thanks to the fact that $\lim _{t \rightarrow 0}\left(E_{\boldsymbol{\alpha}^{\prime}, 1}^{(n)}(t)-1\right)=0$ and the boundedness of $E_{\boldsymbol{\alpha}^{\prime}, 1+\alpha_{1}-\alpha_{j}}^{(n)}(j=$ $2, \ldots, m)$ by Lemma 3.2 for each $n=1,2, \ldots$, the above observation implies

$$
\lim _{t \rightarrow 0}\left(\lambda_{n} t^{\alpha_{1}} E_{\boldsymbol{\alpha}^{\prime}, 1+\alpha_{1}}^{(n)}(t)\right)=0, \quad \forall n=1,2, \ldots
$$

Therefore, (2.3) follows immediately from Lebesgue's dominated convergence theorem.

(c) In order to deal with $\partial_{t} u$, we make use of Lemma 3.3 to obtain

$$
\partial_{t} u(\cdot, t)=-t^{\alpha_{1}-1} \sum_{n=1}^{\infty} \lambda_{n} E_{\boldsymbol{\alpha}^{\prime}, \alpha_{1}}^{(n)}(t)\left(a, \varphi_{n}\right) \varphi_{n} .
$$

Then a similar argument to that for (2.2) indicates

$$
\begin{aligned}
\left\|\partial_{t} u(\cdot, t)\right\|_{L^{2}(\Omega)}^{2} & =t^{2\left(\alpha_{1}-1\right)} \sum_{n=1}^{\infty}\left|\lambda_{n}^{1-\gamma} E_{\boldsymbol{\alpha}^{\prime}, \alpha_{1}}^{(n)}(t)\right|^{2}\left|\lambda_{n}^{\gamma}\left(a, \varphi_{n}\right)\right|^{2} \\
& \leq C^{2} t^{2\left(\alpha_{1}-1\right)} \sum_{n=1}^{\infty}\left(\frac{\left(\lambda_{n} t^{\alpha_{1}}\right)^{1-\gamma}}{1+\lambda_{n} t^{\alpha_{1}}} t^{\alpha_{1}(\gamma-1)}\right)^{2}\left|\lambda_{n}^{\gamma}\left(a, \varphi_{n}\right)\right|^{2} \\
& \leq\left(C\|a\|_{\mathcal{D}\left((-L)^{\gamma}\right)^{\alpha_{1} \gamma-1}}\right)^{2}, \quad 0<t \leq T
\end{aligned}
$$

or (2.4). This implies $\partial_{t} u \in C\left((0, T] ; L^{2}(\Omega)\right)$ immediately.

(d) Finally, to give estimates for $\partial_{t}^{\beta} u$ with $0<\beta<1$ when $\gamma>0$, we employ (2.4) and turn to the definition of the Caputo derivative to obtain

$$
\begin{aligned}
\left\|\partial_{t}^{\beta} u(\cdot, t)\right\|_{L^{2}(\Omega)} & =\frac{1}{\Gamma(1-\beta)}\left\|\int_{0}^{t} \frac{\partial_{s} u(\cdot, s)}{(t-s)^{\beta}} \mathrm{d} s\right\|_{L^{2}(\Omega)} \leq C \int_{0}^{t} \frac{\left\|\partial_{s} u(\cdot, s)\right\|_{L^{2}(\Omega)}}{(t-s)^{\beta}} \mathrm{d} s \\
& \leq C\|a\|_{\mathcal{D}\left((-L)^{\gamma}\right)} \int_{0}^{t} s^{\alpha_{1} \gamma-1}(t-s)^{-\beta} \mathrm{d} s \leq C\|a\|_{\mathcal{D}\left((-L)^{\gamma}\right)^{\alpha_{1} \gamma-\beta}, \quad 0<t \leq T}
\end{aligned}
$$

or (2.5), where the first inequality follows from Minkowski's inequality for integrals. Especially, as long as $\beta \leq \alpha_{1}$, there holds $\alpha_{1} \gamma-\beta>\gamma-1$ and obviously $\partial_{t}^{\beta} v \in L^{\frac{1}{1-\gamma}}\left(0, T ; L^{2}(\Omega)\right)$.

Collecting all the results above, we complete the proof of Theorem 2.1.

Next we turn to the proof of Theorem 2.2, that is, the case of vanishing initial value $a$. To construct an explicit solution, we apply the eigenfunction expansion method. In other words, we seek for a solution to (1.1)-(1.3) of the particular form

$$
u(\cdot, t)=\sum_{n=1}^{\infty} T_{n}(t) \varphi_{n}, \quad 0<t \leq T,
$$

where $\varphi_{n}$ is the $n$-th eigenfunction of $-L$. The substitution of (3.11) into (1.1) yields

$$
\sum_{n=1}^{\infty}\left(\sum_{j=1}^{m} q_{j} \partial_{t}^{\alpha_{j}} T_{n}(t)\right) \varphi_{n}=-\sum_{n=1}^{\infty} \lambda_{n} T_{n}(t) \varphi_{n}+\sum_{n=1}^{\infty}\left(F(\cdot, t), \varphi_{n}\right) \varphi_{n}
$$


Therefore, it is readily seen from the orthogonality of $\left\{\varphi_{n}\right\}$ and the homogeneous initial condition (1.3) that $T_{n}$ satisfies an initial value problem for an ordinary differential equation

$$
\left\{\begin{array}{l}
\sum_{j=1}^{m} q_{j} \partial_{t}^{\alpha_{j}} T_{n}(t)+\lambda_{n} T_{n}(t)=\left(F(\cdot, t), \varphi_{n}\right), \quad 0<t \leq T, \\
T_{n}(0)=0 .
\end{array}\right.
$$

Then it follows from [19, Theorem 4.1] that

$$
T_{n}(t)=\int_{0}^{t} s^{\alpha_{1}-1} E_{\boldsymbol{\alpha}^{\prime}, \alpha_{1}}^{(n)}(s)\left(F(\cdot, t-s), \varphi_{n}\right) \mathrm{d} s,
$$

implying that the solution takes the form of a convolution

$$
u(\cdot, t)=\int_{0}^{t} U(s) F(\cdot, t-s) \mathrm{d} s,
$$

where

$$
U(t) f:=t^{\alpha_{1}-1} \sum_{n=1}^{\infty} E_{\alpha^{\prime}, \alpha_{1}}^{(n)}(t)\left(f, \varphi_{n}\right) \varphi_{n} .
$$

Before proceeding to the proof, we introduce a key lemma for showing Theorem 2.2(a).

Lemma 3.4 (see [3, Theorem 3.2]) The function $t^{\alpha_{1}-1} E_{\boldsymbol{\alpha}^{\prime}, \alpha_{1}}^{(n)}(t)$ is positive for $t>0$.

Proof of Theorem 2.2. Let $F \in L^{p}\left(0, T ; \mathcal{D}\left((-L)^{\gamma}\right)\right)$ with $1 \leq p \leq \infty$ and $0 \leq \gamma \leq 1$. In the sequel, by $C$ we refer to a general positive constant independent of $F$ and $\tau$.

(a) Let $p=2$. According to the expression (3.12)-(3.13), formally we write

$$
\|u(\cdot, t)\|_{\mathcal{D}(-L)}^{2}=\sum_{n=1}^{\infty} \lambda_{n}^{2}\left(\int_{0}^{t} s^{\alpha_{1}-1} E_{\boldsymbol{\alpha}^{\prime}, \alpha_{1}}^{(n)}(s)\left(F(\cdot, t-s), \varphi_{n}\right) \mathrm{d} s\right)^{2} .
$$

Using Young's inequality for convolutions, we estimate

$$
\begin{aligned}
\|u\|_{L^{2}(0, T ; \mathcal{D}(-L))}^{2} & =\sum_{n=1}^{\infty} \lambda_{n}^{2}\left\|\int_{0}^{t} s^{\alpha_{1}-1} E_{\boldsymbol{\alpha}^{\prime}, \alpha_{1}}^{(n)}(s)\left(F(\cdot, t-s), \varphi_{n}\right) \mathrm{d} s\right\|_{L^{2}(0, T)}^{2} \\
& \leq \sum_{n=1}^{\infty}\left(\lambda_{n} \int_{0}^{T} t^{\alpha_{1}-1}\left|E_{\boldsymbol{\alpha}^{\prime}, \alpha_{1}}^{(n)}(t)\right| \mathrm{d} t\right)^{2}\left\|\left(F(\cdot, t), \varphi_{n}\right)\right\|_{L^{2}(0, T)}^{2} .
\end{aligned}
$$

By Lemma 3.4, we can remove the absolute value of $E_{\boldsymbol{\alpha}^{\prime}, \alpha_{1}}^{(n)}(t)$ and apply Lemma 3.3 to derive

$$
\int_{0}^{T} t^{\alpha_{1}-1}\left|E_{\boldsymbol{\alpha}^{\prime}, \alpha_{1}}^{(n)}(t)\right| \mathrm{d} t=\int_{0}^{T} t^{\alpha_{1}-1} E_{\boldsymbol{\alpha}^{\prime}, \alpha_{1}}^{(n)}(t) \mathrm{d} t=T^{\alpha_{1}} E_{\boldsymbol{\alpha}^{\prime}, 1+\alpha_{1}}^{(n)}(T) .
$$

Consequently, we use Lemma 3.2 to conclude

$$
\begin{aligned}
\|u\|_{L^{2}\left(0, T ; H^{2}(\Omega)\right)}^{2} & \leq C^{2}\|u\|_{L^{2}(0, T ; \mathcal{D}(-L))}^{2} \leq C^{2} \sum_{n=1}^{\infty}\left(\lambda_{n} T^{\alpha_{1}} E_{\boldsymbol{\alpha}^{\prime}, 1+\alpha_{1}}^{(n)}(T)\right)^{2}\left\|\left(F(\cdot, t), \varphi_{n}\right)\right\|_{L^{2}(0, T)}^{2} \\
& \leq C^{2} \sum_{n=1}^{\infty}\left\|\left(F(\cdot, t), \varphi_{n}\right)\right\|_{L^{2}(0, T)}^{2}=\left(C\|F\|_{L^{2}(\Omega \times(0, T))}\right)^{2}
\end{aligned}
$$


(b) Fix $\tau \in(0,1]$ arbitrarily. First we give an estimate for (3.13) with $f \in \mathcal{D}\left((-L)^{\gamma}\right)$. Similarly to the proof of Theorem 2.1 we apply Lemma 3.2 to deduce

$$
\begin{aligned}
\|U(t) f\|_{\mathcal{D}((-L) \gamma+1-\tau)}^{2} & =t^{2\left(\alpha_{1}-1\right)} \sum_{n=1}^{\infty}\left|\lambda_{n}^{1-\tau} E_{\boldsymbol{\alpha}^{\prime}, \alpha_{1}}^{(n)}\right|^{2}\left|\lambda_{n}^{\gamma}\left(f, \varphi_{n}\right)\right|^{2} \\
& \leq C^{2} t^{2\left(\alpha_{1}-1\right)} \sum_{n=1}^{\infty}\left(\frac{\left(\lambda_{n} t^{\alpha_{1}}\right)^{1-\tau}}{1+\lambda_{n} t^{\alpha_{1}}} t^{\alpha_{1}(\tau-1)}\right)^{2}\left|\lambda_{n}^{\gamma}\left(f, \varphi_{n}\right)\right|^{2} \\
& \leq\left(C\|f\|_{\mathcal{D}((-L) \gamma)} t^{\alpha_{1} \tau-1}\right)^{2}, \quad 0<t \leq T .
\end{aligned}
$$

Using (3.12) and Minkowski's inequality for integrals, formally we have

$$
\begin{aligned}
\|u(\cdot, t)\|_{\mathcal{D}\left((-L)^{\gamma+1-\tau}\right)} & =\left\|\int_{0}^{t} U(s) F(\cdot, t-s) \mathrm{d} s\right\|_{\mathcal{D}\left((-L)^{\gamma+1-\tau}\right)} \\
& \leq \int_{0}^{t}\|U(s) F(\cdot, t-s)\|_{\mathcal{D}\left((-L)^{\gamma+1-\tau}\right)} \mathrm{d} s \\
& \leq C \int_{0}^{t}\|F(\cdot, t-s)\|_{\mathcal{D}\left((-L)^{\gamma}\right)} s^{\alpha_{1} \tau-1} \mathrm{~d} s, \quad 0<t \leq T .
\end{aligned}
$$

Finally, it follows from Young's inequality for convolutions that

$$
\begin{aligned}
\|u\|_{L^{p}\left(0, T ; \mathcal{D}\left((-L)^{\gamma+1-\tau}\right)\right)} & \leq C\left\|\int_{0}^{t}\right\| F(\cdot, t-s)\left\|_{\mathcal{D}\left((-L)^{\gamma}\right)} s^{\alpha_{1} \tau-1} \mathrm{~d} s\right\|_{L^{p}(0, T)} \\
& \leq C\|F\|_{L^{p}\left(0, T ; \mathcal{D}\left((-L)^{\gamma}\right)\right)} \int_{0}^{T} t^{\alpha_{1} \tau-1} \mathrm{~d} t \leq \frac{C}{\tau}\|F\|_{L^{p}\left(0, T ; \mathcal{D}\left((-L)^{\gamma}\right)\right)} .
\end{aligned}
$$

This completes the verification of (2.7).

(c) Assume $\alpha_{1} p>1$ and fix $\tau \in\left(\frac{1}{\alpha_{1} p}, 1\right]$ arbitrarily. To investigate the asymptotic behavior near $t=0$, we apply Hölder's inequality to (3.14) to see

$$
\|u(\cdot, t)\|_{\mathcal{D}\left((-L)^{\gamma+1-\tau}\right)} \leq C\|F\|_{L^{p}\left(0, t ; \mathcal{D}\left((-L)^{\gamma}\right)\right)}\left(\int_{0}^{t} s^{\left(\alpha_{1} \tau-1\right) p^{\prime}} \mathrm{d} s\right)^{1 / p^{\prime}}
$$

where $p^{\prime}$ is the conjugate number of $p$, i.e. $1 / p+1 / p^{\prime}=1$. Since $\tau>\frac{1}{\alpha_{1} p}$, we see $\left(\alpha_{1} \tau-1\right) p^{\prime}>-1$ and then $\lim _{t \rightarrow 0} \int_{0}^{t} s^{\left(\alpha_{1} \tau-1\right) p^{\prime}} \mathrm{d} s=0$, indicating (2.8) immediately.

As a direct application of Theorems 2.1 2.2, it is straightforward to show the Lipschitz stability of the solution with respect to various coefficients.

Proof of Theorem 2.3. Let $\gamma, \tau \in(0,1], a \in \mathcal{D}\left((-L)^{\gamma}\right)$ and $C>0$ be a general constant which depends only on $a, \mathcal{A}, \mathcal{Q}$ and $\mathcal{U}$. First, a direct application of Theorem 2.1 immediately yields $u \in L^{p}\left(0, T ; H^{2}(\Omega) \cap H_{0}^{1}(\Omega)\right)$ and $\partial_{t}^{\beta} u \in L^{p}\left(0, T ; L^{2}(\Omega)\right)$ for $0<\beta \leq \alpha_{1}$, where we abbreviate $p:=\frac{1}{1-\gamma}$. More precisely, there exists $C>0$ such that

$$
\|u\|_{L^{p}\left(0, T ; H^{2}(\Omega)\right)} \leq C, \quad\left\|\partial_{t}^{\beta} u\right\|_{L^{p}\left(0, T ; L^{2}(\Omega)\right)} \leq C\left(0<\beta \leq \alpha_{1}\right) .
$$


On the other hand, by taking the difference of systems (2.10) and (2.9), it turns out that the system for $v:=\widetilde{u}-u$ reads

$$
\begin{cases}\sum_{j=1}^{m} \widetilde{q}_{j} \partial_{t}^{\widetilde{\alpha}_{j}} v=L_{\widetilde{D}} v+F & \text { in } \Omega \times(0, T] \\ v=0 & \text { on } \partial \Omega \times(0, T], \\ \left.v\right|_{t=0}=0 & \text { in } \Omega,\end{cases}
$$

where

$$
F:=\sum_{j=1}^{m} \widetilde{q}_{j}\left(\partial_{t}^{\alpha_{j}} u-\partial_{t}^{\widetilde{\alpha}_{j}} u\right)+\sum_{j=2}^{m}\left(q_{j}-\widetilde{q}_{j}\right) \partial_{t}^{\alpha_{j}} u+L_{\widetilde{D}-D} u .
$$

Without loss of generality, we assume $\alpha_{1} \geq \widetilde{\alpha}_{1}$, or otherwise we investigate $v:=u-\widetilde{u}$ instead. Therefore, together with $D, \widetilde{D} \in C^{1}(\bar{\Omega})$, we see $F \in L^{p}\left(0, T ; L^{2}(\Omega)\right)$ from (3.15). Now it is straightforward to employ Theorem 2.2(b) to obtain

$$
\|u-\widetilde{u}\|_{L^{p}\left(0, T ; \mathcal{D}\left((-L)^{1-\tau}\right)\right)}=\|v\|_{L^{p}\left(0, T ; \mathcal{D}\left((-L)^{1-\tau}\right)\right)} \leq \frac{C}{\tau}\|F\|_{L^{p}\left(0, T ; L^{2}(\Omega)\right)} .
$$

Especially, if $\gamma \geq \frac{1}{2}$, we see $p=\frac{1}{1-\gamma} \geq 2$ and hence $L^{p}(\Omega \times(0, T)) \subset L^{2}\left(0, T ; L^{2}(\Omega)\right)$. It then follows from Theorem 2.2 (a) that

$$
\|u-\widetilde{u}\|_{L^{2}\left(0, T ; H^{2}(\Omega)\right)} \leq C\|F\|_{L^{2}(\Omega \times(0, T))} \leq C\|F\|_{L^{p}\left(0, T ; L^{2}(\Omega)\right)} .
$$

Therefore, it suffices to dominate $\|F\|_{L^{p}\left(0, T ; L^{2}(\Omega)\right)}$ by the difference of coefficients.

To this end, first it is readily seen from (3.15) that

$$
\begin{aligned}
\|F\|_{L^{p}\left(0, T ; L^{2}(\Omega)\right)} \leq & \sum_{j=1}^{m} \widetilde{q}_{j}\left\|\partial_{t}^{\alpha_{j}} u-\partial_{t}^{\widetilde{\alpha}_{j}} u\right\|_{L^{p}\left(0, T ; L^{2}(\Omega)\right)}+\sum_{j=2}^{m}\left|q_{j}-\widetilde{q}_{j}\right|\left\|\partial_{t}^{\alpha_{j}} u\right\|_{L^{p}\left(0, T ; L^{2}(\Omega)\right)} \\
& +C\|D-\widetilde{D}\|_{C^{1}(\bar{\Omega})}\|u\|_{L^{p}\left(0, T ; H^{2}(\Omega)\right)} \\
\leq & C\left(\sum_{j=1}^{m}\left\|\partial_{t}^{\alpha_{j}} u-\partial_{t}^{\widetilde{\alpha}_{j}} u\right\|_{L^{p}\left(0, T ; L^{2}(\Omega)\right)}+\sum_{j=2}^{m}\left|q_{j}-\widetilde{q}_{j}\right|+\|D-\widetilde{D}\|_{C^{1}(\bar{\Omega})}\right) .
\end{aligned}
$$

To give an estimate for $\partial_{t}^{\alpha_{j}} u-\partial_{t}^{\widetilde{\alpha}_{j}} u$ by $\left|\alpha_{j}-\widetilde{\alpha}_{j}\right|$, we adopt a similar treatment as that in [13, Proposition 1] and decompose it by definition as

$$
\begin{aligned}
\partial_{t}^{\alpha_{j}} u(\cdot, t)-\partial_{t}^{\widetilde{\alpha}_{j}} u(\cdot, t) & =\frac{1}{\Gamma\left(1-\alpha_{j}\right)} \int_{0}^{t} \frac{\partial_{s} u(\cdot, s)}{(t-s)^{\alpha_{j}}} \mathrm{~d} s-\frac{1}{\Gamma\left(1-\widetilde{\alpha}_{j}\right)} \int_{0}^{t} \frac{\partial_{s} u(\cdot, s)}{(t-s)^{\widetilde{\alpha}_{j}}} \mathrm{~d} s \\
& =I_{j}^{1}(\cdot, t)+I_{j}^{2}(\cdot, t),
\end{aligned}
$$

where

$$
\begin{aligned}
& I_{j}^{1}(\cdot, t):=\frac{\Gamma\left(1-\widetilde{\alpha}_{j}\right)-\Gamma\left(1-\alpha_{j}\right)}{\Gamma\left(1-\widetilde{\alpha}_{j}\right)} \partial_{t}^{\alpha_{j}} u(\cdot, t) \\
& I_{j}^{2}(\cdot, t):=\frac{1}{\Gamma\left(1-\widetilde{\alpha}_{j}\right)} \int_{0}^{t}\left\{(t-s)^{-\alpha_{j}}-(t-s)^{-\widetilde{\alpha}_{j}}\right\} \partial_{s} u(\cdot, s) \mathrm{d} s
\end{aligned}
$$


Since $\alpha_{j}, \widetilde{\alpha}_{j} \in[\underline{\alpha}, \bar{\alpha}]$ and the Gamma function is Lipschitz continuous in $[1-\bar{\alpha}, 1-\underline{\alpha}]$, it follows from 3.15 that

$$
\left\|I_{j}^{1}\right\|_{L^{p}\left(0, T ; L^{2}(\Omega)\right)}=\frac{\left|\Gamma\left(1-\widetilde{\alpha}_{j}\right)-\Gamma\left(1-\alpha_{j}\right)\right|}{\Gamma\left(1-\widetilde{\alpha}_{j}\right)}\left\|\partial_{t}^{\alpha_{j}} u\right\|_{L^{p}\left(0, T ; L^{2}(\Omega)\right)} \leq C\left|\alpha_{j}-\widetilde{\alpha}_{j}\right| .
$$

In order to treat $I_{j}^{2}$, we recall the estimate (2.4) for $\partial_{t} u$ and utilize Minkowski's inequality for integrals to deduce

$$
\begin{aligned}
\left\|I_{j}^{2}(\cdot, t)\right\|_{L^{2}(\Omega)} & =\frac{1}{\Gamma\left(1-\widetilde{\alpha}_{j}\right)}\left\|\int_{0}^{t}\left\{(t-s)^{-\alpha_{j}}-(t-s)^{-\widetilde{\alpha}_{j}}\right\} \partial_{s} u(\cdot, s) \mathrm{d} s\right\|_{L^{2}(\Omega)} \\
& \leq \int_{0}^{t}\left|(t-s)^{-\alpha_{j}}-(t-s)^{-\widetilde{\alpha}_{j}}\right|\left\|\partial_{s} u(\cdot, s)\right\|_{L^{2}(\Omega)} \mathrm{d} s \\
& \leq C \int_{0}^{t}\left|(t-s)^{-\alpha_{j}}-(t-s)^{-\widetilde{\alpha}_{j}}\right| s^{\alpha_{1} \gamma-1} \mathrm{~d} s \\
& =C \int_{0}^{t}\left|s^{-\alpha_{j}}-s^{-\widetilde{\alpha}_{j}}\right|(t-s)^{\alpha_{1} \gamma-1} \mathrm{~d} s .
\end{aligned}
$$

Using the mean value theorem, we have

$$
\left|s^{-\alpha_{j}}-s^{-\widetilde{\alpha}_{j}}\right|=|\ln s| s^{-\widehat{\alpha}_{j}(s)}\left|\alpha_{j}-\widetilde{\alpha}_{j}\right|
$$

where $\widehat{\alpha}_{j}(s)$ is a parameter depending on $s$ such that

$$
\min \left\{\alpha_{j}, \widetilde{\alpha}_{j}\right\} \leq \widehat{\alpha}_{j}(s) \leq \max \left\{\alpha_{j}, \widetilde{\alpha}_{j}\right\} \leq \alpha_{1}
$$

by the assumption $\alpha_{1} \geq \widetilde{\alpha}_{1}$. Henceforth, we assume $T>1$ without lose of generality. We prove separately in the cases $0<t \leq 1$ and $1<t \leq T$. First, let $0<t \leq 1$. Then there holds $0<s<1$ and hence

$$
s^{-\widehat{\alpha}_{j}(s)} \leq s^{-\alpha_{1}}=s^{\varepsilon} s^{-\alpha_{1}-\varepsilon},
$$

where $\varepsilon>0$ is sufficiently small such that $\alpha_{1}(1-\gamma)+\varepsilon<1-\gamma$. Since $|\ln s| s^{\varepsilon} \leq C$ for $0<s<1$, we obtain

$$
\begin{aligned}
\left\|I_{j}^{2}(\cdot, t)\right\|_{L^{2}(\Omega)} & \leq C\left|\alpha_{j}-\widetilde{\alpha}_{j}\right| \int_{0}^{t}|\ln s| s^{-\widehat{\alpha}_{j}(s)}(t-s)^{\alpha_{1} \gamma-1} \mathrm{~d} s \\
& \leq C\left|\alpha_{j}-\widetilde{\alpha}_{j}\right| \int_{0}^{t}\left(|\ln s| s^{\varepsilon}\right) s^{-\alpha_{1}-\varepsilon}(t-s)^{\alpha_{1} \gamma-1} \mathrm{~d} s \\
& \leq C\left|\alpha_{j}-\widetilde{\alpha}_{j}\right| t^{-\alpha_{1}(1-\gamma)-\varepsilon}, \quad 0<t \leq 1,
\end{aligned}
$$

where we apply the boundedness of the Beta function $B\left(1-\alpha_{1}-\varepsilon, \alpha_{1} \gamma\right)$ and $\gamma>0$. Second, let $1<t \leq T$. Then it is readily seen that $t-s>1-s$ for $0<s<1$ and $|\ln s| s^{-\widehat{\alpha}_{j}(s)} \leq C$ for $1 \leq s<t$. These observation, together with the inequality (3.19) for $t=1$, indicate

$$
\begin{aligned}
\left\|I_{j}^{2}(\cdot, t)\right\|_{L^{2}(\Omega)} & \leq C\left|\alpha_{j}-\widetilde{\alpha}_{j}\right|\left(\int_{0}^{1}+\int_{1}^{t}\right)|\ln s| s^{-\widehat{\alpha}_{j}(s)}(t-s)^{\alpha_{1} \gamma-1} \mathrm{~d} s \\
& \leq C\left|\alpha_{j}-\widetilde{\alpha}_{j}\right|\left(\int_{0}^{1}|\ln s| s^{-\widehat{\alpha}_{j}(s)}(1-s)^{\alpha_{1} \gamma-1} \mathrm{~d} s+C \int_{1}^{t}(t-s)^{\alpha_{1} \gamma-1} \mathrm{~d} s\right)
\end{aligned}
$$


Multi-Term Time-Fractional Diffusion Equations

$$
\leq C\left|\alpha_{j}-\widetilde{\alpha}_{j}\right|, \quad 1<t \leq T .
$$

The combination of (3.19) and (3.20) immediately yields

$$
\left\|I_{j}^{2}(\cdot, t)\right\|_{L^{2}(\Omega)} \leq C\left|\alpha_{j}-\widetilde{\alpha}_{j}\right| t^{-\alpha_{1}(1-\gamma)-\varepsilon}, \quad 0<t \leq T
$$

and thus $\left\|I_{j}^{2}\right\|_{L^{p}\left(0, T ; L^{2}(\Omega)\right)} \leq C\left|\alpha_{j}-\widetilde{\alpha}_{j}\right|$ because $\alpha_{1}(1-\gamma)+\varepsilon<1-\gamma=1 / p$. Consequently, collecting the estimate (3.18) for $I_{j}^{1}$, we conclude

$$
\begin{aligned}
\|F\|_{L^{p}\left(0, T ; L^{2}(\Omega)\right)} & \leq C\left(\sum_{j=1}^{m}\left\|I_{j}^{1}+I_{j}^{2}\right\|_{L^{p}\left(0, T ; L^{2}(\Omega)\right)}+\sum_{j=2}^{m}\left|q_{j}-\widetilde{q}_{j}\right|+\|D-\widetilde{D}\|_{C^{1}(\bar{\Omega})}\right) \\
& \leq C\left(\sum_{j=1}^{m}\left|\alpha_{j}-\widetilde{\alpha}_{j}\right|+\sum_{j=2}^{m}\left|q_{j}-\widetilde{q}_{j}\right|+\|D-\widetilde{D}\|_{C^{1}(\bar{\Omega})}\right),
\end{aligned}
$$

implying (2.12) and (2.13) with the aid of (3.16) and (3.17) respectively.

\subsection{Proof of Theorem 2.4}

In this subsection, we study the long-time asymptotic behavior of the solution $u$ to (1.1)(1.3) with $F=0$ by a Laplace transform argument. In the sequel, by $C$ we refer to a generic constant which is independent of the initial value $a$ and $u$ but may depend on $d, \Omega, \alpha_{j}, q_{j}$ $(j=1, \ldots, m)$ and the operator $-L$.

Although an explicit representation (3.9) is available in this case, we write the solution in form of

$$
u(\cdot, t)=\sum_{n=1}^{\infty} u_{n}(t) \varphi_{n}, \quad t>0
$$

by use of the eigensystem $\left\{\lambda_{n}, \varphi_{n}\right\}$ of $-L$, where a direct calculation and the orthogonality of $\left\{\varphi_{n}\right\}$ yield

$$
\left\{\begin{array}{l}
\sum_{j=1}^{m} q_{j} \partial_{t}^{\alpha_{j}} u_{n}(t)+\lambda_{n} u_{n}(t)=0, \quad t>0, \quad n=1,2, \ldots \\
u_{n}(0)=\left(a, \varphi_{n}\right),
\end{array}\right.
$$

The proof of Theorem 2.4 relies on the following lemma.

Lemma 3.5 Let $u_{n}(n=1,2, \ldots)$ solve the initial value problem (3.22). Then there exists a constant $C>0$ such that

$$
\left|u_{n}(t)-\frac{q_{m}\left(a, \varphi_{n}\right)}{\lambda_{n} \Gamma\left(1-\alpha_{m}\right) t^{\alpha_{m}}}\right| \leq \frac{C\left|\left(a, \varphi_{n}\right)\right|}{\lambda_{n} t^{\alpha_{m-1}}}, \quad t \gg 1 .
$$

Proof. We abbreviate $a_{n}:=\left(a, \varphi_{n}\right)$ for simplicity. Applying the Laplace transform to (3.22) and using the formula

$$
\mathcal{L}\left(\partial_{t}^{\alpha} f\right)(s)=s^{\alpha} \mathcal{L}(f)(s)-s^{\alpha-1} f(0+),
$$

we are led to the transformed algebraic equation

$$
\mathcal{L}\left(u_{n}\right)(s)=\frac{a_{n}}{w(s)} \sum_{j=1}^{m} q_{j} s^{\alpha_{j}-1}, \quad w(s):=\sum_{j=1}^{m} q_{j} s^{\alpha_{j}}+\lambda_{n} .
$$


Noting that the Laplace transform of $u_{n}$ has a branch point zero, we should cut off the negative part of the real axis so that the function $w(s)$ has no zero in the main sheet of the Riemann surface including its boundaries on the cut. In fact, for $s=r \mathrm{e}^{\mathrm{i} \theta}$, we see that $\sin \left(\alpha_{j} \theta\right)(j=$ $1, \cdots, m)$ have the same signal and thus $\operatorname{Im}(w(s))=\sum_{j=1}^{m} q_{j} r^{\alpha_{j}} \sin \left(\alpha_{j} \theta\right) \neq 0$ since $q_{j}>0$. Therefore, the inverse Laplace transform of $\mathcal{L}\left(u_{n}\right)$ can be represented by an integral on the Hankel path $\mathrm{Ha}(0+)$ (i.e., the loop constituted by a small circle $|s|=\varepsilon$ with $\varepsilon \rightarrow 0$ and by the two borders of the cut negative real axis). Actually, it suffices to consider the following integral

$$
\frac{1}{2 \pi \mathrm{i}} \int_{\mathcal{C}} \mathcal{L}\left(u_{n}\right)(s) \mathrm{e}^{s t} \mathrm{~d} s
$$

and estimate each

$$
H_{\ell}(t ; R):=\int_{\mathcal{C}_{\ell}} \mathcal{L}\left(u_{n}\right)(s) \mathrm{e}^{s t} \mathrm{~d} s, \quad \ell=1, \cdots, 5,
$$

where the loop $\mathcal{C}$ and its partitions $\mathcal{C}_{\ell}(\ell=1, \ldots, 5)$ are illustrated in Figure 2

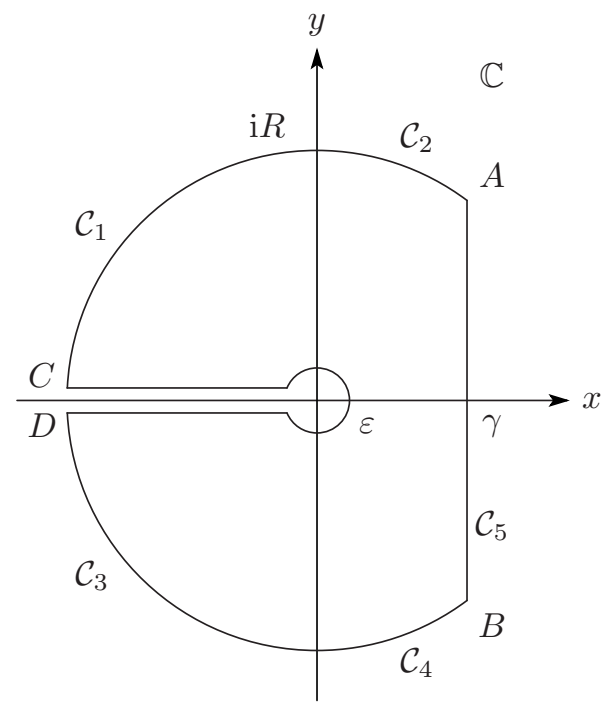

Figure 2. The loop $\mathcal{C}$ and its partition.

For $H_{1}(t ; R)$, noting that $|s|=R>1$ and using a change of variable, we have

$$
\begin{aligned}
\left|H_{1}(t ; R)\right| & =\left|\int_{\mathcal{C}_{1}} \mathcal{L}\left(u_{n}\right)(s) \mathrm{e}^{s t} \mathrm{~d} s\right| \leq C\left|a_{n}\right| \int_{\pi / 2}^{\pi} R^{\alpha_{m}} \mathrm{e}^{R t \cos \theta} \mathrm{d} \theta \\
& =C\left|a_{n}\right| R^{\alpha_{m}} \int_{-1}^{0} \frac{\mathrm{e}^{R t \eta}}{\sqrt{1-\eta^{2}}} \mathrm{~d} \eta, \quad t>0 .
\end{aligned}
$$

Furthermore, we break up the above integral in $[-1,0]$ into two parts and calculate their bounds respectively as

$$
\begin{aligned}
R^{\alpha_{m}} \int_{-1}^{0} \frac{\mathrm{e}^{R t \eta}}{\sqrt{1-\eta^{2}}} \mathrm{~d} \eta & =R^{\alpha_{m}}\left(\int_{-1}^{-1 / 2}+\int_{-1 / 2}^{0}\right) \frac{\mathrm{e}^{R t \eta}}{\sqrt{1-\eta^{2}}} \mathrm{~d} \eta \\
& \leq R^{\alpha_{m}} \mathrm{e}^{-R t / 2} \int_{-1}^{-1 / 2} \frac{\mathrm{d} \eta}{\sqrt{1-\eta^{2}}}+C R^{\alpha_{m}} \int_{-1 / 2}^{0} \mathrm{e}^{R t \eta} \mathrm{d} \eta
\end{aligned}
$$




$$
\leq C R^{\alpha_{m}} \mathrm{e}^{-R t / 2}+C R^{\alpha_{m}-1} \frac{1-\mathrm{e}^{-R t / 2}}{t} \rightarrow 0 \quad \text { as } R \rightarrow \infty, t>0 .
$$

Therefore, for any $t>0$, we see that $H_{1}(t ; R) \rightarrow 0$ as $R \rightarrow \infty$. Similarly to the calculation of $H_{1}(t ; R)$, we have $H_{3}(t ; R) \rightarrow 0$ as $R \rightarrow \infty$ for any $t>0$. On the other hand, since $R \cos \theta \leq \gamma$ for all $\theta \in\left[\theta_{R}, \pi / 2\right]$ where $\theta_{R}$ denotes the argument of point $A$, we have

$$
\begin{aligned}
\left|H_{2}(t ; R)\right| & \leq C\left|a_{n}\right| R^{\alpha_{m}} \int_{\theta_{R}}^{\pi / 2} \mathrm{e}^{R t \cos \theta} \mathrm{d} \theta \leq C\left|a_{n}\right| R^{\alpha_{m}} \mathrm{e}^{\gamma t}\left(\frac{\pi}{2}-\theta_{R}\right) \\
& =C\left|a_{n}\right| R^{\alpha_{m}} \mathrm{e}^{\gamma t}\left(\frac{\pi}{2}-\arccos \frac{1}{R}\right) \rightarrow 0 \quad \text { as } R \rightarrow \infty .
\end{aligned}
$$

Therefore, since $w(s)$ has no zero in the main sheet of the Riemann surface including the boundaries on the cut, the integral in (3.24) vanishes. By Fourier-Mellin formula (see e.g. 27]), we have

$$
u_{n}(t)=\lim _{M \rightarrow \infty} \frac{1}{2 \pi \mathrm{i}} \int_{\gamma-\mathrm{i} M}^{\gamma+\mathrm{i} M} \mathcal{L}\left(u_{n}\right)(s) \mathrm{e}^{s t} \mathrm{~d} s=\frac{1}{2 \pi \mathrm{i}} \int_{\operatorname{Ha}(\varepsilon)} \mathcal{L}\left(u_{n}\right)(s) \mathrm{e}^{s t} \mathrm{~d} s .
$$

Here the integral is taken on the segment from $\gamma-\mathrm{i} M$ to $\gamma+\mathrm{i} M$, and $\mathrm{Ha}(\varepsilon)$ denotes the Hankel path in $\mathbb{C}$ defined as

$$
\operatorname{Ha}(\varepsilon):=\{s \in \mathbb{C} ; \arg s= \pm \pi,|s| \geq \varepsilon\} \cup\{s \in \mathbb{C} ;-\pi \leq \arg s \leq \pi,|s|=\varepsilon\} .
$$

By a similar argument as above, we find

$$
\frac{1}{\Gamma\left(1-\alpha_{m}\right) t^{\alpha_{m}}}=\lim _{M \rightarrow \infty} \frac{1}{2 \pi \mathrm{i}} \int_{\gamma-\mathrm{i} M}^{\gamma+\mathrm{i} M} s^{\alpha_{m}-1} \mathrm{e}^{s t} \mathrm{~d} s=\frac{1}{2 \pi \mathrm{i}} \int_{\mathrm{Ha}(\varepsilon)} s^{\alpha_{m}-1} \mathrm{e}^{s t} \mathrm{~d} s .
$$

It is now straightforward to show that the contribution from the Hankel path $\mathrm{Ha}(\varepsilon)$ as $\varepsilon \rightarrow 0$ is provided by

$$
\begin{aligned}
& u_{n}(t)-\frac{q_{m} a_{n}}{\lambda_{n} \Gamma\left(1-\alpha_{m}\right) t^{\alpha_{m}}}=a_{n} \int_{0}^{\infty} H\left(r, \lambda_{n}\right) \mathrm{e}^{-r t} \mathrm{~d} r, \quad \text { where } \\
& H\left(r, \lambda_{n}\right):=-\frac{1}{\pi} \operatorname{Im}\left\{\left.\left(\frac{1}{w(s)} \sum_{j=1}^{m} q_{j} s^{\alpha_{j}-1}-\frac{q_{m}}{\lambda_{n}} s^{\alpha_{m}-1}\right)\right|_{s=r \mathrm{e} \mathrm{e}^{\mathrm{i} \pi}}\right\} .
\end{aligned}
$$

To give the desired estimate (3.23), we observe that $|w(s)| \geq C \lambda_{n}$ as long as $r=|s| \leq \varepsilon_{0} \lambda_{n}$, where $\varepsilon_{0}>0$ is sufficiently small. This indicates

$$
\begin{aligned}
\left|H\left(r, \lambda_{n}\right)\right| & \leq\left|\frac{\lambda_{n} \sum_{j=1}^{m-1} q_{j} s^{\alpha_{j}-1}-\sum_{j=1}^{m} q_{j} q_{m} s^{\alpha_{j}+\alpha_{m}-1}}{\lambda_{n}\left(\sum_{j=1}^{m} q_{j} s^{\alpha_{j}}+\lambda_{n}\right)}\right| \\
& \leq \frac{C\left|a_{n}\right|}{\lambda_{n}}\left(\sum_{j=1}^{m-1}|s|^{\alpha_{j}-1}+\sum_{j=1}^{m}|s|^{\alpha_{j}+\alpha_{m}-1}\right), \quad \forall|s| \leq \varepsilon_{0} \lambda_{n} .
\end{aligned}
$$

Meanwhile, for any $s=r \mathrm{e}^{ \pm \mathrm{i} \pi}$ with $r \geq \varepsilon_{0} \lambda_{n}$, we know that

$$
\left|H\left(r, \lambda_{n}\right)\right| \leq \frac{\sum_{j=1}^{m-1} q_{j} r^{\alpha_{j}-1}}{\left|\operatorname{Im} \sum_{j=1}^{m} q_{j} s^{\alpha_{j}}\right|}+\frac{\sum_{j=1}^{m} q_{j} q_{m} r^{\alpha_{j}+\alpha_{m}-1}}{\lambda_{n}\left|\operatorname{Im} \sum_{j=1}^{m} q_{j} s^{\alpha_{j}}\right|} \leq C .
$$


Using these estimates, we break up the integral in (3.25) into two parts and give respective bounds as

$$
\begin{aligned}
\left|\int_{0}^{\varepsilon_{0} \lambda_{n}} H\left(r, \lambda_{n}\right) \mathrm{e}^{-r t} \mathrm{~d} r\right| & \leq \frac{C}{\lambda_{n}} \int_{0}^{\infty}\left(\sum_{j=1}^{m-1} r^{\alpha_{j}-1}+\sum_{j=1}^{m} r^{\alpha_{j}+\alpha_{m}-1}\right) \mathrm{e}^{-r t} \mathrm{~d} r \\
& \leq \frac{C}{\lambda_{n}}\left(\sum_{j=1}^{m-1} \frac{1}{t^{\alpha_{j}}}+\sum_{j=1}^{m} \frac{1}{t^{\alpha_{j}+\alpha_{m}}}\right) \\
\left|\int_{\varepsilon_{0} \lambda_{n}}^{\infty} H\left(r, \lambda_{n}\right) \mathrm{e}^{-r t} \mathrm{~d} r\right| & \leq C \int_{\varepsilon_{0} \lambda_{n}}^{\infty} \mathrm{e}^{-r t} \mathrm{~d} r=\frac{C}{t \mathrm{e}^{\varepsilon_{0} \lambda_{n} t}} \leq \frac{C}{\lambda_{n} t^{2}}
\end{aligned}
$$

Collecting the above two estimates, we obtain (3.23) for sufficiently large $t$.

Proof of Theorem 2.4. Let $u$ take the form of (3.21) which solves (1.1)-(1.3) with $a \in L^{2}(\Omega)$ and $F=0$, and fix any $T>0$ sufficiently large. For all $t \geq T$, it immediately follows from Lemma 3.5 and the eigenfunction expansion that

$$
\begin{aligned}
\left\|u(\cdot, t)-\frac{(-L)^{-1}\left(q_{m} a\right)}{\Gamma\left(1-\alpha_{m}\right) t^{\alpha_{m}}}\right\|_{H^{2}(\Omega)} & \leq C\left\|\sum_{n=1}^{\infty}\left(u_{n}(t)-\frac{q_{m}\left(a, \varphi_{n}\right)}{\lambda_{n} \Gamma\left(1-\alpha_{m}\right) t^{\alpha_{m}}}\right) \varphi_{n}\right\|_{\mathcal{D}(-L)} \\
& =C\left(\sum_{n=1}^{\infty}\left|\lambda_{n} u_{n}(t)-\frac{q_{m}\left(a, \varphi_{n}\right)}{\Gamma\left(1-\alpha_{m}\right) t^{\alpha_{m}}}\right|^{2}\right)^{1 / 2} \\
& \leq \frac{C}{t^{\alpha_{m}-1}}\left(\sum_{n=1}^{\infty}\left|\left(a, \varphi_{n}\right)\right|^{2}\right)^{1 / 2}=\frac{C\|a\|_{L^{2}(\Omega)}}{t^{\alpha_{m-1}}}
\end{aligned}
$$

implying $u \in C\left([T, \infty) ; H^{2}(\Omega) \cap H_{0}^{1}(\Omega)\right)$. On the other hand, since Theorem 2.1(a) guarantees $u \in C\left([0, T] ; L^{2}(\Omega)\right) \cap C\left((0, T] ; H^{2}(\Omega) \cap H_{0}^{1}(\Omega)\right)$, the proof is finished by combining the regularity results in the finite and infinite time spans.

Remark 3.1 If some $q_{j_{0}}$ is negative, then we cannot obtain the asymptotic estimate for the solution $u$ of the initial-boundary value problem (1.1)-(1.3). In fact, for some $n \in \mathbb{N}$ sufficiently large, we study the following problem

$$
\begin{cases}\partial_{t}^{1 / 2} u(x, t)-3 \lambda_{n} \partial_{t}^{1 / 4} u(x, t)=L u(x, t), & x \in \Omega, t>0 \\ u(x, t)=0, & x \in \partial \Omega, t>0 . \\ u(x, 0)=a_{n} \varphi_{n}=\left(a, \varphi_{n}\right) \varphi_{n}, & x \in \Omega,\end{cases}
$$

where $\left(\lambda_{n}, \varphi_{n}\right)$ is the $n$-th pair in the eigensystem of the elliptic operator $-L$, and $a \in L^{2}(\Omega)$. The Laplace transform of the solution reads

$$
\mathcal{L}(u)(s)=\frac{a_{n}}{w(s)}\left(s^{-1 / 2}-3 \lambda_{n} s^{-3 / 4}\right) \varphi_{n}, \quad w(s):=s^{1 / 2}-3 \lambda_{n} s^{1 / 4}+\lambda_{n} .
$$

We see that $\{s ; w(s)=0\}$ is a finite set with all of the zero points having finite multiplicity in the main sheet of the Riemann surface, and there is no zero point on the negative part of the real axis since $\lambda_{n}$ is sufficiently large. Furthermore, we can prove that there exist zeros of $w(s)$ having positive real parts. In fact, obviously

$$
r_{ \pm}:=\frac{3 \lambda_{n} \pm \sqrt{9 \lambda_{n}^{2}-4 \lambda_{n}}}{2}>0
$$


solves $w\left(r_{ \pm}\right)=0$, and we have

$$
w^{\prime}\left(r_{ \pm}\right)=\frac{1}{2} r_{ \pm}^{-1 / 2}-\frac{3 \lambda_{n}}{4} r_{ \pm}^{-3 / 4} \neq 0
$$

Note that

$$
\frac{1}{2 \pi \mathrm{i}} \int_{\mathcal{C}} \mathcal{L}(u)(s) \mathrm{e}^{s t} \mathrm{~d} s=\sum \operatorname{Res}\left\{\mathcal{L}(u)(s) \mathrm{e}^{s t}, \mathcal{C}\right\},
$$

where $\mathcal{C}$ is defined in Figure 2 Res $\{f, \mathcal{C}\}$ denotes the residue of function $f$ in the domain enclosed by $\mathcal{C}$, and the sum is taken over all the poles of $\mathcal{L}(u)(s) \mathrm{e}^{s t}$ in this domain. Repeating the argument in the proof of Lemma 3.5, we deduce

$$
u(t)=\lim _{M \rightarrow \infty} \frac{1}{2 \pi \mathrm{i}} \int_{\gamma-\mathrm{i} M}^{\gamma+\mathrm{i} M} \mathcal{L}(u)(s) \mathrm{e}^{s t} \mathrm{~d} s=\sum \operatorname{Res}\left\{\mathcal{L}(u)(s) \mathrm{e}^{s t}\right\}+\frac{1}{2 \pi \mathrm{i}} \int_{\mathrm{Ha}(0+)} \mathcal{L}(u)(s) \mathrm{e}^{s t} \mathrm{~d} s .
$$

Here the sum is taken over all the poles of $\mathcal{L}(u)(s) \mathrm{e}^{s t}$ lying on the left-hand side of the line $\{z=\gamma+\mathrm{i} M ; M \in \mathbb{R}\}$ with $\gamma>r_{+}$, and there are only finite terms in this summation since $w(s)$ only has finite number of zero points including multiplicity in the main sheet of the Riemann surface cutting of the negative axis. We can easily see that

$$
\operatorname{Res}\left\{\left.\left(\mathcal{L}(u)(s) \mathrm{e}^{s t}\right)\right|_{s=r_{ \pm}}\right\}=\frac{r_{ \pm}^{-1 / 2}-3 \lambda_{n} r_{ \pm}^{-3 / 4}}{w^{\prime}\left(r_{ \pm}\right)} \mathrm{e}^{r_{ \pm} t} \varphi_{n}
$$

Of course $\mathrm{e}^{r_{ \pm} t}$ tend to infinity as $t \rightarrow \infty$ since $r_{ \pm}>0$, indicating that the asymptotic behavior in Theorem 2.4 does not hold for this case.

\section{Concluding Remarks}

We summarize this paper by providing several concluding remarks. Concerning the initialboundary value problem (1.1)-(1.3) for multi-term time-fractional diffusion equations, we mainly investigate the well-posedness and the long-time asymptotic behavior of the solution, which turn out to be mostly parallel to those of the single-term prototype. On the basis of the representation of solutions and a careful analysis of multinomial Mittag-Leffler functions, we succeed in dominating the solutions by the initial value $a$ and the source term $F$. Although uniqueness and stability also follow from the maximum principle developed in [18, we carry out various estimates so that regularity and short-time asymptotic behaviors of the solutions are directly connected with the regularity of $a$ and $F$ (see Theorems 2.1 2.2). Furthermore, in Theorem 2.3 we establish the Lipschitz stability of the solution with respect to $\alpha_{j}, q_{j}$ and the diffusion coefficient, which is not only important by itself but also applicable to the corresponding inverse coefficient problem when treated by a minimization approach (see [13. Theorem 5]).

Simultaneously, we also obtain an extended version of [26, Corollary 2.6] in Theorem 2.4. which asserts that, if the solution does not vanish identically, then its decay rate cannot exceed $t^{-\alpha_{m}}$, where $\alpha_{m}$ is the minimum order of fractional time-derivative. It is a remarkable property of fractional diffusion equations because the classical diffusion equation admits non-zero solutions decaying exponentially. This characterizes the slow diffusion in contrast to the classical one. 
In the formulation of the initial-boundary value problem, we emphasize that the coefficients $q_{j}$ of the time derivatives are positive constants because this assumption is obligatory not only to acquire explicit solutions but also to apply the Laplace transform in time, which are essential in the discussions of well-posedness and asymptotic behavior, respectively. On the other hand, if $q_{j}$ are space-dependent, then explicit solutions are not available so that one should rely on a fixed point argument for the unique existence of solution, and the improvement of regularity in space is strictly less than 2 orders (see [4, Theorem 2]). On the other hand, if some $q_{j_{0}}$ is negative, then one may construct a counterexample in which the asymptotic property fails (see Remark 3.1).

However, in view of practical applications and theoretical interests, the linear non-symmetric diffusion equation with positive variable coefficients of Caputo derivatives in time can be regarded as a more feasible model equation than that we have studied in the current paper, but it will be definitely more challenging. Though still under consideration, we expect to establish parallel results for this more generalized case.

Acknowledgement The second author appreciates the invaluable discussions with Professor William Rundell, Professor Raytcho Lazarov, Dr. Lihua Zuo and Mr. Zhi Zhou (Texas A\&M University).

\section{References}

[1] E.E. Adams, L.W. Gelhar, Field study of dispersion in a heterogeneous aquifer 2. Spatial moments analysis, Water Resources Res. 28 (1992) 3293-3307.

[2] R.A. Adams, Sobolev Spaces, Academic Press, New York, 1975.

[3] E. Bazhlekova, Properties of the fundamental and the impulse-response solutions of multi-term fractional differential equations, in: V. Kiryakova (Ed.), Complex Analysis and Applications '13 (Proc. Intern. Conf., Sofia, 2013), Bulg. Acad. Sci. Sofia, 2013, pp. 55-64.

[4] S. Beckers, M. Yamamoto, Regularity and unique existence of solution to linear diffusion equation with multiple time-fractional derivatives, in: K. Bredies, C. Clason, K. Kunisch, G. von Winckel (Eds.), Control and Optimization with PDE Constraints, Birkhäuser, Basel, 2013, pp. 45-56.

[5] C. Berge, Principles of Combinatorics, Academic Press, New York, 1971.

[6] M. Ginoa, S. Cerbelli, H.E. Roman, Fractional diffusion equation and relaxation in complex viscoelastic materials, Phys. A 191 (1992) 449-453.

[7] R. Gorenflo, Y. Luchko, P.P. Zabrejko, On solvability of linear fractional differential equations in Banach spaces, Frac. Calc. Appl. Anal. 2 (1999) 163-176.

[8] R. Gorenflo, F. Mainardi, Fractional calculus: Integral and differential equations of fractional order, in: A. Carpinteri, F. Mainardi (Eds.), Fractals and Fractional Calculus in Continuum Mechanics, Springer-Verlag, New York, 1997, pp. 223-276.

[9] Y. Hatano, N. Hatano, Dispersive transport of ions in column experiments: an explanation of long-tailed profiles, Water Resources Res. 34 (1998) 1027-1033.

[10] H. Jiang, F. Liu, I. Turner, K. Burrage, Analytical solutions for the multi-term time-space Caputo-Riesz fractional advection-diffusion equations on a finite domain, J. Math. Anal. Appl. 389 (2012) 1117-1127.

[11] B. Jin, R. Lazarov, Z. Zhou, Error estimates for a semidiscrete finite element method for fractional order parabolic equations, SIAM J. Numer. Anal. 51 (2013) 445-466.

[12] A.A. Kilbas, H.M. Srivastava, J.J. Trujillo, Theory and Applications of Fractional Differential Equations, Elsevier, Amsterdam, 2006.

[13] G. Li, D. Zhang, X. Jia, M. Yamamoto, Simultaneous inversion for the space-dependent diffusion coefficient and the fractional order in the time-fractional diffusion equation, Inverse Problems 29 (2013) 065014. 
[14] F. Liu, P. Zhuang, V. Anh, I. Turner, K. Burrage, Stability and convergence of the difference methods for the space-time fractional advection-diffusion equation, Appl. Math. Comput. 191 (2007) 12-20.

[15] Y. Luchko, Initial-boundary value problems for the generalized time-fractional diffusion equation, in: Proceedings of 3rd IFAC Workshop on Fractional Differentiation and Its Applications (FDA08), Ankara, Turkey, 05-07 November 2008.

[16] Y. Luchko, Maximum principle for the generalized time-fractional diffusion equation, J. Math. Anal. Appl. 351 (2009) 218-223.

[17] Y. Luchko, Some uniqueness and existence results for the initial-boundary value problems for the generalized time-fractional diffusion equation, Comput. Math. Appl. 59 (2010) 1766-1772.

[18] Y. Luchko, Initial-boundary-value problems for the generalized multi-term time-fractional diffusion equation, J. Math. Anal. Appl. 374 (2011) 538-548.

[19] Y. Luchko, R. Gorenflo, An operational method for solving fractional differential equations with the Caputo derivatives, Acta Math. Vietnam, 24 (1999) 207-233.

[20] M.M. Meerschaert, C. Tadjeran, Finite difference approximations for fractional advection-dispersion flow equations, J. Comput. Appl. Math. 172 (2004) 65-77.

[21] F. Mainardi, A. Mura, G. Pagnini, R. Gorenflo, Time-fractional diffusion of distributed order, J. Vib. Control 14 (2008) 1267-1290.

[22] R.R. Nigmatullin, The realization of the generalized transfer equation in a medium with fractal geometry, Phys. Stat. Sol. B 133 (1986) 425-430.

[23] A. Pazy, Semigroups of Linear Operators and Applications to Partial Differential Equations, SpringerVerlag, Berlin, 1983.

[24] I. Podlubny, Fractional Differential Equations, Academic Press, San Diego, 1999.

[25] J. Prüss, Evolutionary Integral Equations and Applications, Birkhauser, Basel, 1993.

[26] K. Sakamoto, M. Yamamoto, Initial value/boundary value problems for fractional diffusion-wave equations and applications to some inverse problems, J. Math. Anal. Appl. 382 (2011) 426-447.

[27] J. L. Schiff, The Laplace Transform: Theory and Applications, Springer-Verlag, New York, 1999.

[28] S.G. Samko, A.A. Kilbas, O.I. Marichev, Fractional Integrals and Derivatives, Gordon and Breach Science Publishers, Philadelphia, 1993. 